\title{
Effects of moderately fast shellular rotation on adiabatic oscillations ${ }^{\star}$
}

\author{
J. C. Suárez ${ }^{1,2, \star \star}$, M. J. Goupil ${ }^{2}$, and P. Morel ${ }^{3}$ \\ ${ }^{1}$ Instituto de Astrofísica de Andalucía (CSIC), CP 3004, Granada, Spain \\ e-mail: jcsuarez@iaa.es \\ 2 LESIA, UMR 8109, Observatoire de Paris-Meudon, France \\ 3 Observatoire de la Côte d'Azur, Nice, France
}

Received 9 September 2005 / Accepted 11 November 2005

\section{ABSTRACT}

We investigate adiabatic oscillations for $\delta$ Scuti star models, taking a moderate rotation velocity (around $100 \mathrm{~km} \mathrm{~s}^{-1}$ ) into account. The resulting oscillation frequencies include corrections for rotation up to second order in the rotation rate including those of near degeneracy. Effects of either a uniform rotation or a rotation profile assuming local angular momentum conservation of the form $\Omega=\Omega(r)$ on oscillation frequencies are compared. As expected, important differences (around $3 \mu \mathrm{Hz}$ ) are obtained in the $g$ and mixed-mode regions. For higher-frequency $p$ modes, differences range between $1 \mu \mathrm{Hz}$ and $3 \mu \mathrm{Hz}$. Such differences are likely to be detectable with future space missions such as COROT, where precisions in frequency around $0.5 \mu \mathrm{Hz}$ are expected to be reached.

Key words. stars: variables: $\delta$ Sct - stars: rotation - stars: oscillations - stars: interiors - stars: fundamental parameters - stars: evolution

\section{Introduction}

Intermediate mass stars are characterized by a convective core and a radiative envelope. As representative of such stars, $\delta$ Scuti stars are located in the lower part of the Cepheid instability strip, with spectral types from A2 to F0. Such pulsating stars seem particularly suitable for determining the extent of the convective core and internal rotation rates, and thus for better understanding the hydrodynamical processes taking place in stellar interiors. Particularly, balance between rotationally induced turbulence and meridional circulation generates the mixing of chemicals and redistribution of angular momentum (Zahn 1992), which then affects the rotation profile and the evolution of the star. It is expected that intermediate mass stars do not rotate uniformly as a solid body. Zahn (1992) proposed that, as a result of strong anisotropic turbulence, the stellar rotation profile is shellular, i.e. the star is divided in differentially rotating concentric shells.

The $\delta$ Scuti stars can be found in two main evolutionary stages: on the main sequence, where they burn hydrogen in their convective cores, and before the sub-giant phase, when burning hydrogen in shells. They can be evolved enough to present a strong gradient of chemical composition, produced by

* Appendices A-C are only available in electronic form at http://www.edpsciences.org

$\star \star$ Associate researcher at institute (2), with financial support from the Spanish «Consejería de Innovación, Ciencia y Empresa» from the «Junta de Andalucía» local government. their shrinking convective core. These types of structures generate particular modes, showing a dual behavior. These modes are known as mixed modes, which behave like pressure modes ( $p$ modes) at surface and like gravity modes ( $g$ modes) toward the center (Christensen-Dalsgaard 1998). Indeed, they can penetrate sufficiently deep toward the center of the star and at the same time show amplitudes at the surface that are large enough to be detected. The existence of such modes can thus be very important when investigating deep interiors of stars.

In past decades, there have been extensive efforts to develop observational seismology for $\delta$ Scuti stars, for instance the world-wide observational campaigns (Breger 2000; Handler 2000) or the STEPHI network (Michel et al. 2000). However, several observational aspects of the pulsating behavior within the instability strip are not fully understood (see Templeton et al. 1997). Due to the complexity of the oscillation spectra of $\delta$ Scuti stars, the problem of identifying modes remains unsolved. As $\delta$ Scuti stars are commonly fast rotators $(100<$ $v \sin i<200 \mathrm{~km} \mathrm{~s}^{-1}$ ), additional uncertainties to their already complex oscillation spectra arise from the effect of rapid rotation. Rotation alters the internal structure of a star through a modified hydrostatic balance and, probably more important, through mixing caused by circulation and/or instabilities induced by rotation (Zahn 1992; Maeder \& Meynet 2000; Heger et al. 2000). Furthermore the simple characteristic pattern of symmetric multiplets split by rotation is broken. In the framework of a perturbation analysis, second-order effects induce strong asymmetries in the splitting of multiplets (Saio 1981; 
Dziembowski \& Goode 1992, hereafter DG92) and frequency shifts that cannot be neglected even for radial modes (Soufi et al. 1995).

In DG92, the authors propose a second-order formalism to study the eigenfunctions for the Sun and $\delta$ Scuti stars, with both radial and latitudinal rotation, which takes into account the effects of near degeneracy up to the first order in the rotation rate. For medium-high rotators, like $\delta$ Scuti stars, the effects of near degeneracy up to the second and third orders cannot be neglected (see Dziembowski \& Goode 1992; Goupil et al. 2000). This last point is included in a complete third-order formalism given by Soufi et al. (1998, hereafter SGD98). Near degeneracy affects the asymmetry of multiplets, making the interpretation of observed oscillation spectra even more difficult. Based on this approach, assuming a uniform rotation for the sake of simplicity, theoretical and quantitative studies on the oscillations have followed of rotating $\delta$ Scuti stars (Goupil et al. 2000; Goupil \& Talon 2002; Daszyńska-Daszkiewicz et al. 2002; Pamyatnykh 2003), of solar-like stars (Dziembowski $\&$ Goupil 1998; Goupil et al. 2004), and of $\beta$ Cephei stars (Daszyńska-Daszkiewicz et al. 2003). SGD98's work has been recently revisited and applied to the study of oscillations of $\beta$ Cephei stars (Karami et al. 2005, in press).

The present work focuses on the magnitude of the effect of shellular rotation on the adiabatic oscillations of a $1.8 M_{\odot}$ star. In order to avoid possible interferences between the third-order effects of rotation on oscillations and those coming from shellular rotation, only second-order terms are considered. To do so, we built a numerical code (Suárez 2002) taking a complete formalism up to the second order into account in the presence of a shellular rotation $\Omega=\Omega(r)$.

Theoretical oscillation spectra computed for two different models of $\delta$ Scuti star are compared: one model was evolved assuming uniform rotation (global conservation of the angular momentum), and another model was evolved assuming a shellular rotation (local conservation of the angular momentum). The impact of a shellular rotation on oscillation frequencies is discussed in the frame of the future space experiment COROT (launch in 2006).

The paper is organized as follows: Sect. 2 briefly recalls the basis of the second-order perturbation formalism of nondegenerate oscillation frequencies. Section 3 describes neardegeneracy theory as implemented in our oscillation code. In Sect. 4 the adopted methodology is detailed. Section 5 discusses the results and finally conclusions are given in Sect. 6.

\section{Non-degenerate oscillation frequencies corrected for rotation effects}

Following DG92, when Coriolis and centrifugal forces are considered, the eigenfrequency $\omega$ and associated eigenfunctions $\boldsymbol{\xi}$ must satisfy the following oscillation equation:

$\mathcal{L}_{0} \boldsymbol{\xi}-\rho_{0} \hat{\omega}^{2} \boldsymbol{\xi}-2 \rho_{0} \hat{\omega} \Omega \boldsymbol{K} \boldsymbol{\xi}+\left(\mathcal{L}_{2}-\rho_{2} \hat{\omega}^{2}\right) \boldsymbol{\xi}=0$,

where $\hat{\omega}=\omega+m \Omega$ and $\boldsymbol{K}=\mathbf{i} \boldsymbol{e}_{z} \times$ (SGD98, Eq. (22)). The linear operators $\mathcal{L}_{0}$ and $\mathcal{L}_{2}$ correspond to $\boldsymbol{L}_{0}$ and $\boldsymbol{L}_{2}$ defined in SGD98 (Eqs. (23) and (24)), respectively. The mean density of the pseudo rotating model is represented by $\rho_{0}$. Likewise, its perturbation by the non-spherically symmetric component of the centrifugal force is given by the following expansion in Legendre polynomials

$\rho_{2}=p_{22}(r) P_{2}(\cos \theta)$,

where $p_{22}(r)$ is defined in SGD98 (Eq. (15)). With appropriate boundary conditions, Eq. (1) forms an eigenvalue problem (Appendix A). The eigenfunction $\xi$ is written as

$\boldsymbol{\xi}=\boldsymbol{\xi}_{0}+\boldsymbol{\xi}_{1}$

where $\xi_{0}$ and $\xi_{1}$ correspond to the non perturbed and first-order perturbed eigenfunctions, respectively, defined as:

$$
\begin{aligned}
\boldsymbol{\xi}_{0}= & r\left[y_{01} Y_{\ell}^{m} \boldsymbol{e}_{r}+z_{0} \boldsymbol{\nabla}_{\mathrm{H}} Y_{\ell}^{m}\right] \\
\boldsymbol{\xi}_{1}= & \frac{2 m \bar{\Omega}}{\omega_{0}} r\left[y_{1} Y_{\ell}^{m} \boldsymbol{e}_{r}+z_{1} \boldsymbol{\nabla}_{\mathrm{H}} Y_{\ell}^{m}\right. \\
& \left.+\tau_{\ell+1} \boldsymbol{e}_{r} \times \boldsymbol{\nabla}_{\mathrm{H}} Y_{\ell+1}^{m}+\hat{\tau}_{\ell-1} \boldsymbol{e}_{r} \times \boldsymbol{\nabla}_{\mathrm{H}} Y_{\ell-1}^{m}\right],
\end{aligned}
$$

where the notations are the same as in SGD98. In these expressions, $y_{01}$ and $z_{0}$ represent the normalized radial and horizontal components, respectively, of the eigenfunction for the fluid displacement (Unno et al. 1989). Similarly, the first-order corrections are represented by $y_{1}$ and $z_{1}$, respectively (see Appendix A.1).

The eigenfrequency associated with each mode is thus labeled with the subscripts $n, \ell, m$ representing, respectively, the radial order, degree, and azimuthal order of the corresponding spherical harmonics. The temporal dependence is chosen with the form $\exp (\mathrm{i} \omega t)$, so that prograde modes correspond to $m<0$. Following DG92, oscillation frequencies are obtained by means of a perturbative method taking up to second order effects of rotation into account. Equilibrium and oscillating quantities are expanded with respect to $\epsilon=\Omega \sigma / \omega$ and $\mu=\Omega / \sigma$, where $\sigma$ is the dimensionless mode frequency defined as $\sigma=\omega /\left(G M / R^{3}\right)^{1 / 2} ; R$ and $M$ represent the stellar radius and the mass of the model respectively.

Ignoring the resonant interaction due to near degeneracy in this section, the frequency of a given rotationally split multiplet of degree $\ell$ and radial order $n$ can be written as:

$\omega_{m}=\omega_{0}+\omega_{1, m}+\omega_{2, m}$,

where the subscripts $n, \ell$ have been omitted. The $\omega_{m}$ frequencies are obtained by means of a perturbative method. The zeroth-order contribution, $\omega_{0}$, represents the mode frequency with all effects of rotation ignored except that of the horizontally averaged centrifugal force in the equilibrium model (later, we will abusively refer to it as the "non perturbed" frequency). The other two terms, $\omega_{1, m}$ and $\omega_{2, m}$, represent the first- and second-order corrections.

A shellular rotation is considered with a rotation profile defined as

$\Omega(r)=\bar{\Omega}\left[1+\eta_{0}(r)\right]$, 
where $\bar{\Omega}$ represents the rotation frequency at the stellar surface. In this context, the first- and second-order frequency corrections are written as:

$\omega_{1, m}=m \bar{\Omega}\left(C_{\mathrm{L}}-1-J_{0}\right)$

$\omega_{2, m}=\frac{\bar{\Omega}^{2}}{\omega_{0}}\left(D_{0}+m^{2} D_{1}\right)$.

The Ledoux constant, $C_{\mathrm{L}}$, that determines the usual equidistant splitting valid in the limit of slow rotation, is given by the wellknown integral expression

$C_{\mathrm{L}}=\frac{1}{I_{0}} \int_{0}^{R}\left[2 y_{01} z_{0}+z_{0}^{2}\right] \rho_{0} r^{4} \mathrm{~d} r$

where $I_{0}$ is the term of inertia given by:

$I_{0}=\int_{0}^{R}\left[y_{01}^{2}+\Lambda z_{0}^{2}\right] \rho_{0} r^{4} \mathrm{~d} r$

with $\Lambda=\ell(\ell+1)$. In Eq. (8), $J_{0}$ represents an additional contribution in the case of shellular rotation and is given by:

$J_{0}=\frac{1}{I_{0}} \int_{0}^{R} \eta_{0}(r)\left[y_{01}^{2}+\Lambda z_{0}^{2}-2 y_{01} z_{0}-z_{0}^{2}\right] \rho_{0} r^{4} \mathrm{~d} r$.

The second-order coefficients $D_{0}$ and $D_{1}$ in Eq. (9) take into account the non-spherically symmetrical distortion due to the centrifugal force. The symmetry of split multiplets is broken by the $m^{2}$ dependency (see Eq. (9)). For later use, we also rewrite these coefficients in Saio's notation:

$D_{0} \equiv X_{1}+X_{2} \quad D_{1} \equiv Y_{1}+Y_{2}$.

Comparing Eq. (9) with the formulation given in DG92, the following relations are obtained:

$\omega_{0}\left(\frac{\bar{\Omega}}{\omega_{0}}\right)^{2}\left(X_{1}+m^{2} Y_{1}\right)=\omega_{2}^{\mathrm{T}}+\omega_{2}^{\mathrm{I}}+\frac{\omega_{1}^{2}}{2 \omega_{0}}+\omega_{2}^{\mathrm{P}}$

for $X_{1}$ and $Y_{1}$ and

$\omega_{0}\left(\frac{\bar{\Omega}}{\omega_{0}}\right)^{2}\left(X_{2}+m^{2} Y_{2}\right)=\omega_{2}^{\mathrm{D}}$

for $X_{2}$ and $Y_{2}$. The analytic expressions for $X_{i}$ and $Y_{i}$ are given in Appendix B. The terms $\omega_{2}^{\mathrm{T}}, \omega_{2}^{\mathrm{I}}$, and $\omega_{2}^{\mathrm{P}}$, defined by Eqs. (16)-(19) in DG92, represent the different contributions to second-order frequency corrections in the absence of degeneracy effects and, as in SGD98, include the effect of the symmetrical component of the centrifugal force on the equilibrium model: $\omega_{2}^{\mathrm{P}}$ and $\omega_{2}^{\mathrm{T}}$ represent the poloidal and toroidal components of the frequency, respectively, obtained from the first-order correction to the eigenfunction; $\omega_{2}^{\mathrm{D}}$ corrects for the effects of the centrifugal force on the stellar structure.

\section{Near-degenerate oscillation frequencies}

The perturbation method presented in the previous section assumes that the non-perturbed eigenmode can be represented with one single spherical harmonic. This is no longer valid in the case of near-degenerate frequencies, i.e. when two or more frequencies are close to each other $\left(\omega_{n l m} \sim \omega_{n l m^{\prime}}\right)$. Consequently, the perturbation method must be modified in order to include corrections for near degeneracy as done in the next sections.

\subsection{The oscillation equation in presence of degeneracy}

The formalism is similar for 2 or 3 near-degenerate modes. For the sake of simplicity, it is illustrated below for two modes $a$ and $b$. The $a$ and $b$ subscripts represent $(n, \ell, m)_{a}$ and $(n, \ell, m)_{b}$, respectively. For near degenerate mode $a$ and mode $b$, it is convenient to define:

$$
\begin{aligned}
\bar{\omega}_{0} & =\frac{\omega_{0, a}+\omega_{0, b}}{2} \\
\delta \omega_{0} & =\omega_{0, a}-\omega_{0, b} .
\end{aligned}
$$

The eigenfrequency and the eigenfunction of a near-degenerate mode are then assumed to be of the form:

$$
\begin{aligned}
\omega & =\bar{\omega}_{0}+\tilde{\omega}_{1}+\tilde{\omega}_{2} \\
\boldsymbol{\xi} & =\sum_{j=a, b} \alpha_{j}\left(\xi_{0, j}+\xi_{1, j}\right)
\end{aligned}
$$

First and second-order corrections to the eigenfrequency in the presence of near degeneracy are represented by $\tilde{\omega}_{1}$ and $\tilde{\omega}_{2}$, respectively; $\xi_{0, j}$ and $\xi_{1, j}$ are the non perturbed and firstorder perturbed eigenfunctions defined in Eqs. (4) and (5), respectively.

Let us now replace $\omega$ and $\boldsymbol{\xi}$ by Eqs. (18) and (19) in the oscillation equation (Eq. (1)). Perturbation is then performed keeping in mind that $\delta \omega_{0}$ is small, of first or second order in the rotation rate, i.e. $\delta \omega_{0} \sim O(\Omega)$ or $\delta \omega_{0} \sim O\left(\Omega^{2}\right)$. Projecting onto the non-perturbed eigenfunctions $\boldsymbol{\xi}_{0, a}$ and $\boldsymbol{\xi}_{0, b}$, the following system is obtained:

$\sum_{j=a, b}\left[\mathcal{M}_{j k}^{(1)}+\mathcal{M}_{j k}^{(2)}+\frac{\delta \omega_{0}}{2} \mathcal{I}_{-1}+\frac{\delta \omega_{0}^{2}}{8 \bar{\omega}_{0}} \mathcal{I}_{1}\right] \alpha_{j}=0$

for $k=a, b$. In this equation we have defined the unit matrix $\mathcal{I}_{1}$ and also the matrix $\mathcal{I}_{-1}$ as:

$\mathcal{I}_{-1}=\left(\begin{array}{rr}1 & 0 \\ 0 & -1\end{array}\right)$

The first- and second-order matrix $\mathcal{M}_{j k}$ terms are given by:

$\mathcal{M}_{j k}^{(1)}=\left(\begin{array}{lr}-\tilde{\omega}_{1}+\omega_{1, j} & \omega_{1, j \mathrm{k}} \\ \omega_{1, k j} & -\tilde{\omega}_{1}+\omega_{1, k}\end{array}\right)$
$\mathcal{M}_{j k}^{(2)}=\left(\begin{array}{lr}-\tilde{\omega}_{2}+\mu_{j} & \mu_{j k} \\ \mu_{k j} & -\tilde{\omega}_{2}+\mu_{k}\end{array}\right)$,

where we have defined:

$\mu_{j}=-\frac{\tilde{\omega}_{1}}{\omega_{0, j}}\left(\frac{\tilde{\omega}_{1}}{2}-\omega_{1, j}\right)+\omega_{2, j}^{\mathrm{D}}+\omega_{2, j}^{\mathrm{P}}+\omega_{2, j}^{\mathrm{T}}+\omega_{2, j}^{\mathrm{I}}$

$\mu_{j k}=\tilde{\omega}_{1} \frac{\omega_{1, j \mathrm{k}}}{\omega_{0, k}}+\omega_{2, j \mathrm{k}}^{\mathrm{D}}+\omega_{2, j \mathrm{k}}^{\mathrm{P}}+\omega_{2, j \mathrm{k}}^{\mathrm{T}}+\omega_{2, j \mathrm{k}}^{\mathrm{I}}$.

Terms with one single subscript are not affected by near degeneracy and are defined in Sect. 2. Coupling terms for degeneracy are included in $\omega_{1, j \mathrm{k}}$ and $\mu_{j k}$; they are defined with doublesubscript terms and their definitions and detailed expressions are given in Appendix C. 


\subsection{Selection rules}

Corrections for near degeneracy exist only for modes with degrees $\ell$ and azimuthal orders $m$ fulfilling specific selection rules:

- Analysis of $\omega_{1, a b}$ (Eq. (C.7)) shows that frequency corrections for near degeneracy already arise at first order whenever the modes have same degree $\ell$ and same azimuthal order $m$, i.e. $\ell_{a}=\ell_{b}$ and $m_{a}=m_{b}$. The modes then differ only by their radial orders $n_{a} \neq n_{b}$.

- Near degeneracy affects second-order frequency corrections of near-degenerate frequencies (see Appendix C) whenever $\ell_{a}=\ell_{b}$ or $\ell_{a}=\ell_{b} \pm 2$ and $m_{a}=m_{b}$. These selection rules are also obtained in the case of the compound third-order treatment given by SGD98. Equation (20) is then solved for $j=a$ and $k=b$ for each case allowed by the selection rules.

For two near-degenerate modes, $a$ and $b$, the proximity in frequency is expected to be less than or equal to the rotation frequency of the stellar model $\left(\left|\omega_{a}-\omega_{b}\right| \lesssim \Omega\right.$ or $\left.\Omega^{2} / \omega_{a}\right)$ depending on the degrees and azimuthal orders of the modes. This estimate may vary with the nature of the modes $(g, p$, or mixed modes). For stellar models of interest here, numerical applications reveal that many modes have near-degenerate frequencies.

\subsection{First-order near degeneracy}

In this case $\delta \omega_{0}$ is $O(\Omega)$. Only modes $a$ and $b$ with $\ell_{a}=\ell_{b}$ modes are affected. Such a situation generally concerns only a few modes that are in avoided crossing. We find the first-order correction to the frequency as the condition for the existence of non-trivial solutions of Eq. (20):

$\left(-\tilde{\omega}_{1}+\omega_{1, a}+\frac{\delta \omega_{0}}{2}\right)\left(-\tilde{\omega}_{1}+\omega_{1, b}-\frac{\delta \omega_{0}}{2}\right)-\mathcal{W}_{1, a b}=0$

where

$\mathcal{W}_{1, a b}=\omega_{1, a b} \omega_{1, b a}$.

In the case of two degenerate modes, this system is equivalent to Eqs. (59), (60) in DG92. Conditions for non-trivial solutions give the first-order frequency corrections in presence of near degeneracy

$\tilde{\omega}_{1}=\frac{\omega_{1, a}+\omega_{1, b}}{2} \pm \sqrt{\mathcal{H}_{1, a b}}$

with

$\mathcal{H}_{1, a b}=\left(\frac{\omega_{1, a}-\omega_{1, b}}{2}+\frac{\delta \omega_{0}}{2}\right)^{2}+\mathcal{W}_{1, a b}$

Note that, in the case of negligible near degeneracy, that is, when $4 \omega_{1, a b}^{2} \ll\left(\omega_{1, a}-\omega_{1, b}-\delta \omega_{0}\right)^{2}$, the non-degenerate $\omega_{1, a}$ and $\omega_{1, b}$ frequencies are retrieved. The second-order neardegenerate frequency correction is again obtained as the condition for a non-trivial solution of Eq. (20):

$\left(v_{a}+\mu_{a}-\tilde{\omega}_{2}+\frac{\delta \omega_{0}^{2}}{8 \bar{\omega}_{0}}\right)\left(v_{b}+\mu_{b}-\tilde{\omega}_{2}+\frac{\delta \omega_{0}^{2}}{8 \bar{\omega}_{0}}\right)-\mathcal{W}_{2, a b}=0$ where we have defined

$\mathcal{W}_{2, a b}=\left(\omega_{1, a b}+\mu_{a b}\right)\left(\omega_{1, b a}+\mu_{b a}\right)$

and

$v_{b}=\left(-\tilde{\omega}_{1}+\omega_{1, b}-\frac{\delta \omega_{0}}{2}\right)$

with $\tilde{\omega}_{1}$ given by Eq. (26). Equation (28) provides two firstorder solutions, $\tilde{\omega}_{1}^{+}$and $\tilde{\omega}_{1}^{-}$. Four possible solutions for $\tilde{\omega}_{2}$ are thus obtained. Although all of them are mathematically valid, only two have a physical meaning. Each second-order solution $\tilde{\omega}_{2}=\tilde{\omega}_{2}^{+}$and $\tilde{\omega}_{2}^{-}$is associated to one and only one first-order solution $\tilde{\omega}_{1}^{-}$and $\tilde{\omega}_{1}^{+}$, respectively. We then obtain:

$\tilde{\omega}_{2}=\left(\frac{v_{b}+v_{a}}{2}+\frac{\mu_{b}+\mu_{a}}{2}+\frac{\delta \omega_{0}^{2}}{8 \bar{\omega}_{0}}\right) \pm \sqrt{\mathcal{H}_{2, a b}^{(1)}}$,

where

$\mathcal{H}_{2, a b}^{(1)}=h(\nu, \mu)_{a, b}^{2}+\mathcal{W}_{2, a b}$

with

$h(v, \mu)_{a, b}=\frac{\left(v_{a}+\mu_{a}\right)-\left(v_{b}+\mu_{b}\right)}{2}$.

\subsection{Second-order near degeneracy}

In this case, $\delta \omega_{0}$ is $O\left(\Omega^{2}\right), \tilde{\omega}_{1}=\omega_{1}$, and $\omega_{1, j k}=0$ in Eq. (24). The quantity $\mu_{j}$ in Eq. (25) is then $\omega_{2 j}$, as defined in Eq. (9). Only modes with $\ell_{a}=\ell_{b} \pm 2$ are concerned. The analytic expression of second-order frequency corrections is obtained as the condition for existence of non-trivial solutions for

$\sum_{j=a, b} \alpha_{j}\left[\mathcal{M}_{j k}^{(2)}+\frac{\delta \omega_{0}}{2} \mathcal{I}_{-1}\right]=0$

We then obtain

$\tilde{\omega}_{2}=\left(\frac{\mu_{b}+\mu_{a}}{2}\right) \pm \sqrt{\mathcal{H}_{2, a b}^{(2)}}$

where

$\mathcal{H}_{2, a b}^{(2)}=\left(\frac{\mu_{b}-\mu_{a}}{2}-\frac{\delta \omega_{0}}{2}\right)^{2}+\mu_{a b} \mu_{b a}$.

When near degeneracy is negligible, $4 \mu_{a b} \mu_{b a} \ll\left(\mu_{a}-\mu_{b}+\right.$ $\left.\delta \omega_{0}\right)^{2}$, and the non-degenerate solutions $\mu_{a}=\omega_{2 a}$ and $\mu_{b}=\omega_{2 b}$ are respectively retrieved.

Our numerical computations suggest that for rapid enough rotations, every pair of modes having $\Delta \ell= \pm 2$ with radial orders $n$ and $n-1$, respectively, should present frequencies that are near enough to be coupled. For pairs with $\ell=0,2$, coupling is more significant for high radial order modes.

\section{Evolutionary models}

Equilibrium stellar models have been computed with the evolution code CESAM. Following the approach described in Kippenhahn \& Weigert (1990), equilibrium models are constructed by modifying the stellar structure equations so as to 
include the spherical symmetric contribution of the centrifugal acceleration, by means of an effective gravity:

$g_{\mathrm{eff}}=g-\mathcal{A}_{\mathrm{c}}(r)$,

where $g$ is the local gravity component at a radial distance $r$

$g=G \frac{m(r)}{r^{2}}$

with $m(r)$ the spherical mass at radius $r, G$ the gravitational constant. And $\mathcal{A}_{\mathrm{c}}(r)$ corresponds to the centrifugal acceleration at radius $r$ :

$\mathcal{A}_{\mathrm{c}}(r)=\frac{2}{3} r \Omega^{2}(r)$.

This spherically symmetric contribution of the rotation does not change the shape of the hydrostatic equilibrium equation. This is not fully representative of the structure of a rotating star.

First, the non-spherical component of the deformation of the star is not considered. However, its effects are included through a perturbation in the oscillation equations (see previous sections). Second, rotationally-induced mixing and transport of angular momentum can significantly modify the rotation profile (Talon et al. 1997; Maeder \& Meynet 2000; Heger et al. 2000; Denissenkov \& Tout 2000; Palacios et al. 2003). As these processes are not included in our models, we consider here two illustrative cases when prescribing $\Omega(r)$.

No mass loss is considered at any evolutionary stage, that is, the total angular momentum is assumed to be conserved. In this framework, the two assumptions for the transport of the angular momentum are: 1) either instantaneous transport of angular momentum in the whole star (global conservation), which thus yields a uniform rotation; or 2) for sake of simplicity and illustrative purpose, local conservation of the angular momentum (shellular rotation). Formally, the local conservation of angular momentum between two instants $\left(t_{2}>t_{1}\right)$ can be expressed as:

$\frac{2}{3} r_{1}^{2}(m) \Omega_{1}(m)=\frac{2}{3} r_{2}^{2}(m) \Omega_{2}(m)$,

where $m$ is the Lagrangian abscissa coordinate. Rotation in the convective core is considered to be rigid. As shown by Eq. (39), gravity is modified by the radial term of the centrifugal acceleration $\mathcal{A}_{\mathrm{c}}$ (see Eq. (41)). In Fig. 1, $\mathcal{A}_{\mathrm{c}}$ is displayed as a function of the radius for both cases (uniform and shellular rotation). The radial distance $r=0.1 R$ corresponds to a local maximum that is the limit of the convective core. This implies a modification of the local density, particularly in such regions.

In Fig. 2, evolutionary tracks for a $1.8 M_{\odot}$ model computed with three different assumptions: uniform rotation $\left(J_{G}\right)$, shellular rotation $\left(J_{L}\right)$, and absence of rotation $(\Omega=0)$. Two evolutionary stages are labeled: the zero-age main sequence ( $A, A_{J}$ and $A_{L}$ models) and on the main sequence ( $B, B_{J}$ and $B_{L}$ models). Fundamental stellar parameters of these models are given in Table 1. During the main sequence evolution of the star, the rotation profile along the stellar radius mainly results from 1) the contraction of the core and 2) the expansion of the outer layers. Then the local moment of inertia of contracting shells of a given elementary mass decreases. Therefore, the

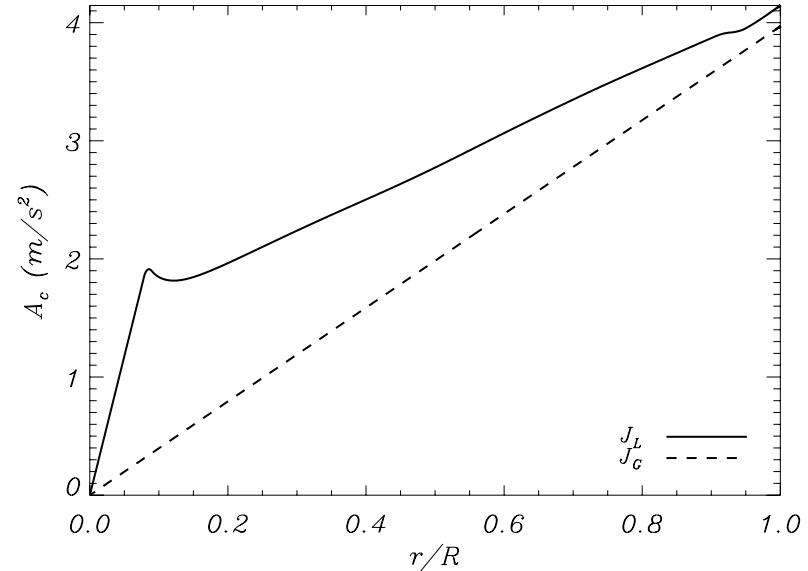

Fig. 1. Radial component of the centrifugal acceleration for a differentially rotating stellar model with $J_{L}$ (continuous line) and a uniform rotating one with $J_{G}$ (dashed line) considered in the present study. These quantities are displayed for a $1.8 M_{\odot}$ model, aged $1050 \mathrm{Myr}$ with a photometric radius of $R=2.27 R_{\odot}$.

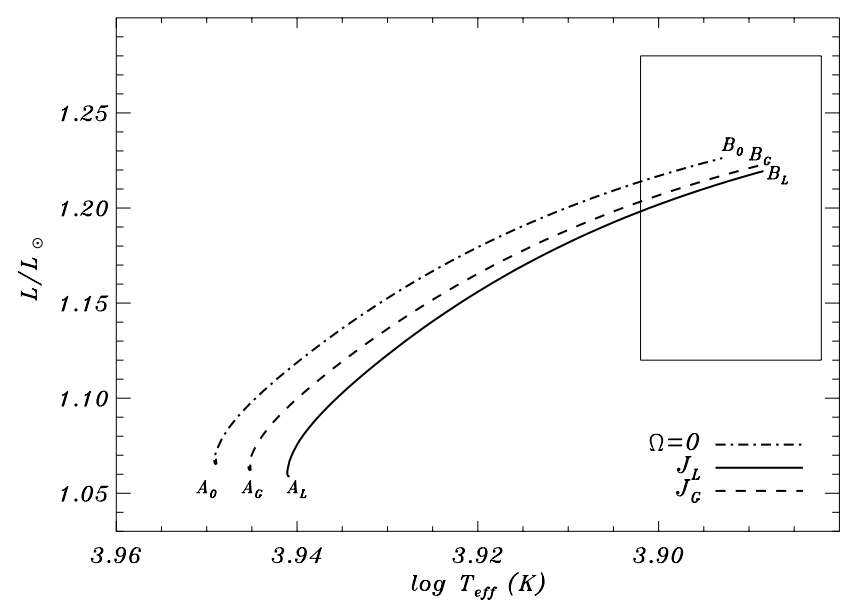

Fig. 2. Evolutionary tracks of $1.8 M_{\odot}$ models representative of a typical $\delta$ Scuti star. The three tracks correspond to: a uniform rotating model (dashed line), a shellular rotating model (continuous line), and finally a non-rotating model (dash-dotted line). The box represents typical observational errors for $\delta$ Scuti stars.

Table 1. Characteristics of the three $1.8 M_{\odot}$ models considered in Fig. 2. From left to right, $\Omega_{\mathrm{s}}$ represents the surface rotation frequency, $T_{\text {eff }}$ the effective temperature, $X_{\mathrm{c}}$ the central hydrogen fraction, and $\bar{\rho}$ the mean density. Two evolutionary stages are considered: the zero age main sequence $(A)$ and a main sequence age of $1050 \mathrm{Myr}(B)$. A solar metallicity, a mixing length parameter of $\alpha_{M L}=1.614$, and an overshooting parameter of $d_{\mathrm{ov}}=0.2$ have been assumed.

\begin{tabular}{cccccc}
\hline \hline Model & $\begin{array}{c}\Omega_{\mathrm{s}} \\
(\mu \mathrm{Hz})\end{array}$ & $\begin{array}{c}R \\
\left(R_{\odot}\right)\end{array}$ & $\begin{array}{c}\log T_{\text {eff }} \\
(\mathrm{K})\end{array}$ & $X_{\mathrm{c}}$ & $\begin{array}{c}\bar{\rho} \\
\left(\mathrm{g} \mathrm{cm}^{-3}\right)\end{array}$ \\
\hline$A_{\mathrm{L}}$ & 15.485 & 1.824 & 3.870 & 0.726 & 7.308 \\
$A_{G}$ & 10.902 & 1.800 & 3.873 & 0.726 & 7.555 \\
$A_{0}$ & 0 & 1.795 & 3.876 & 0.726 & 7.927 \\
$B_{\mathrm{L}}$ & 9.980 & 2.271 & 3.888 & 0.318 & 2.839 \\
$B_{G}$ & 9.757 & 2.275 & 3.888 & 0.315 & 2.827 \\
$B_{0}$ & 0 & 2.242 & 3.892 & 0.312 & 3.939 \\
\hline
\end{tabular}




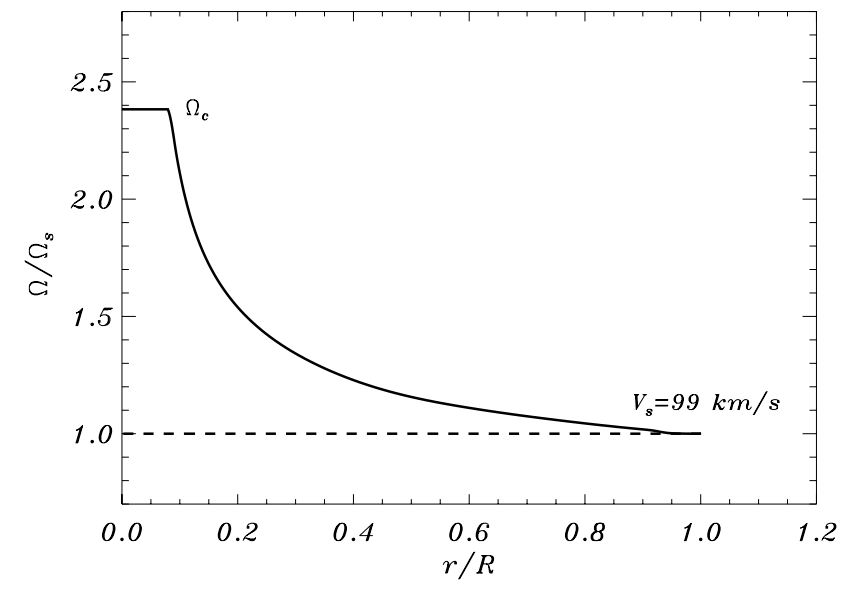

Fig. 3. Rotation frequency through the star normalized to its surface value. The rotation profiles correspond to the $B_{\mathrm{L}}$ model (curved line, shellular rotation) and the $B_{G}$ model (horizontal line, uniform rotation), respectively. Models are built with the same surface rotation frequency $\Omega_{\mathrm{S}}=9.98 \mu \mathrm{Hz}$. For more details see Sect. 4 .

local rotation frequency of those shells must increase in order to conserve the angular momentum locally. Consequently, the rotation frequency toward the surface decreases during the evolution of the star (see Fig. 3). This figure shows a strong gradient of the rotation frequency toward the center. At a height of $r=0.2 R$ the rotation rate is $\Omega=1.5 \Omega_{\mathrm{s}}$ with $\Omega_{\mathrm{s}}$ the surface rotation rate. In our model, this means a rotation frequency around $14.97 \mu \mathrm{Hz}$ at $r=0.2 R$, while a rotation frequency of $23.78 \mu \mathrm{Hz}$ is obtained at the limit of the convective core $(r \sim 0.1 R)$. Oscillation modes with amplitude in these regions (typically $g$ and mixed modes) are expected to be sensitive to shellular rotation. In practice, observations provide the surface velocity (most often through the $v \sin i$ parameter). In order to reproduce such conditions, models $B_{L}$ and $B_{G}$ were evolved so as to obtain the same rotation velocity at the surface. The first one $\left(B_{\mathrm{L}}\right)$ was evolved considering the assumption of local conservation of the angular momentum. The second one $\left(B_{G}\right)$ has been evolved under the assumption of uniform rotation (global conservation of the angular momentum).

\section{Effects of shellular rotation on oscillation frequencies}

Oscillation frequencies of a rotating star are obtained from Eq. (6). For the sake of clarity and brevity, the following nomenclature will be used: $\omega_{0}^{L}$ and $\omega_{0}^{G}$ for zeroth order frequencies, $\omega_{1}^{L}$ and $\omega_{1}^{G}$ for first-order frequency corrections, and finally $\omega_{2}^{L}$ and $\omega_{2}^{G}$ for second-order frequency corrections. The $L$ and $G$ superscripts follow the same nomenclature adopted for the models $B_{L}$ and $B_{G}$. Similarly, differences in frequency for a given mode (given $n, \ell, m$ subscripts) between two models will be denoted as $\Delta \omega_{j}=\omega_{j}^{L}-\omega_{j}^{G}$ for $j=0,1,2$ associated with zeroth-order frequencies, first- and second-order frequency corrections, respectively.

It is found that the behavior of frequency corrections does not significantly change for low $\ell$ degree values $(\ell \leq 3)$ when a uniform or a shellular rotation profile is assumed. We therefore focus on $\ell=1$ modes in this section.

In the following sections, the analysis and discussion of the effect of shellular rotation on oscillation frequencies include very high-order $p$ modes. The reader should notice that such modes present a low probability of being observed (even from space) due to their expected small amplitudes. Even so, from a theoretical point of view, investigation of the frequencies of these modes - i.e. with well-defined (asymptotic) behavior - is helpful for the overall interpretation of the oscillation spectrum.

\subsection{Zeroth-order frequency corrections}

Perturbing and linearizing the hydrodynamical equations about a pseudo-rotating model yield an eigenvalue system: see Eq. (A.5) in Appendix A for more details. Our numerical oscillation code is adapted to solve this system, with the boundary conditions specified in Eqs. (A.16)-(A.19) and a radial shellular rotation profile included in Eq. (7). The resolution of this system yields the zeroth order frequency $\omega_{0}$. The use of pseudo-rotating models described in Sect. 4 provides zeroth-order eigenfrequencies that include the effects of the spherically symmetric perturbation of pressure, density, and gravitational potential. These perturbations are induced by the spherically symmetric component of the centrifugal acceleration (see SGD98). Eigenmodes of this system are still $m$ degenerate.

Oscillation frequencies are thus computed for the $B_{\mathrm{L}}$ and $B_{G}$ models described in Sect. 4. In order to quantify the effect of shellular rotation on zeroth-order frequencies, $\Delta \omega_{0}=\omega_{0}^{L}-\omega_{0}^{G}$ differences are calculated. These differences are shown for $\ell=$ 1 modes in Fig. 6 (solid line) as a function of the radial order $n$ of the mode. As can be seen, the effect of shellular rotation on zeroth-order frequencies is very small at low frequencies and increases with the radial order $n$.

For high radial order $p$ modes $(n \geq 12)$, the differences $\Delta \omega_{0}$ are larger than differences from higher-order corrections $\Delta \omega_{1}, \Delta \omega_{2}$, (Sects. 3.3, 3.4) and hence become the most sensitive to the effect of shellular rotation. Numerical tests show that the differences $\Delta \omega_{0}$ mainly come from the differences in the pseudo rotating models, $B_{\mathrm{L}}, B_{G}$; the contribution of the rotation profile (either uniform or shellular ) in the oscillation equations is negligible.

As an order of magnitude, for $\ell=0, n \sim 10-15$ modes, we consider the slope, $v_{a}(v=\omega / 2 \pi)$, of the variation of the differences $\left(v_{0, n+1, \ell=0,0}-v_{0, n, \ell=0,0}\right)$ with the radial order $n$ (in the asymptotic approximation). We find numerically that $\Delta v_{a}=$ $\left(v_{a}^{L}-v_{a}^{R}\right) \sim 0.175 \mu \mathrm{Hz}$ for $v_{a} \sim 47 \mu \mathrm{Hz}$; i.e. a relative difference $\Delta v_{a} / v_{a} \sim 3.7 \times 10^{-3}$. This difference can be accounted for by the fact that our $B_{L}$ and $B_{G}$ models have slightly different radii, $\Delta R / R=\left(R^{L}-R^{G}\right) / R^{L}=-1.76 \times 10^{-3}$ (see Table 1$)$. This induces a contribution to $\Delta v_{a}$ that can be quantified as:

$\frac{\Delta v_{a}}{v_{a}}=-\frac{3}{2} \frac{\Delta R}{R} \sim 2.6 \times 10^{-3}$

Hence, in the present case, quite large differences, $\Delta \omega_{0}$, at high frequency come from structural properties of the two models 

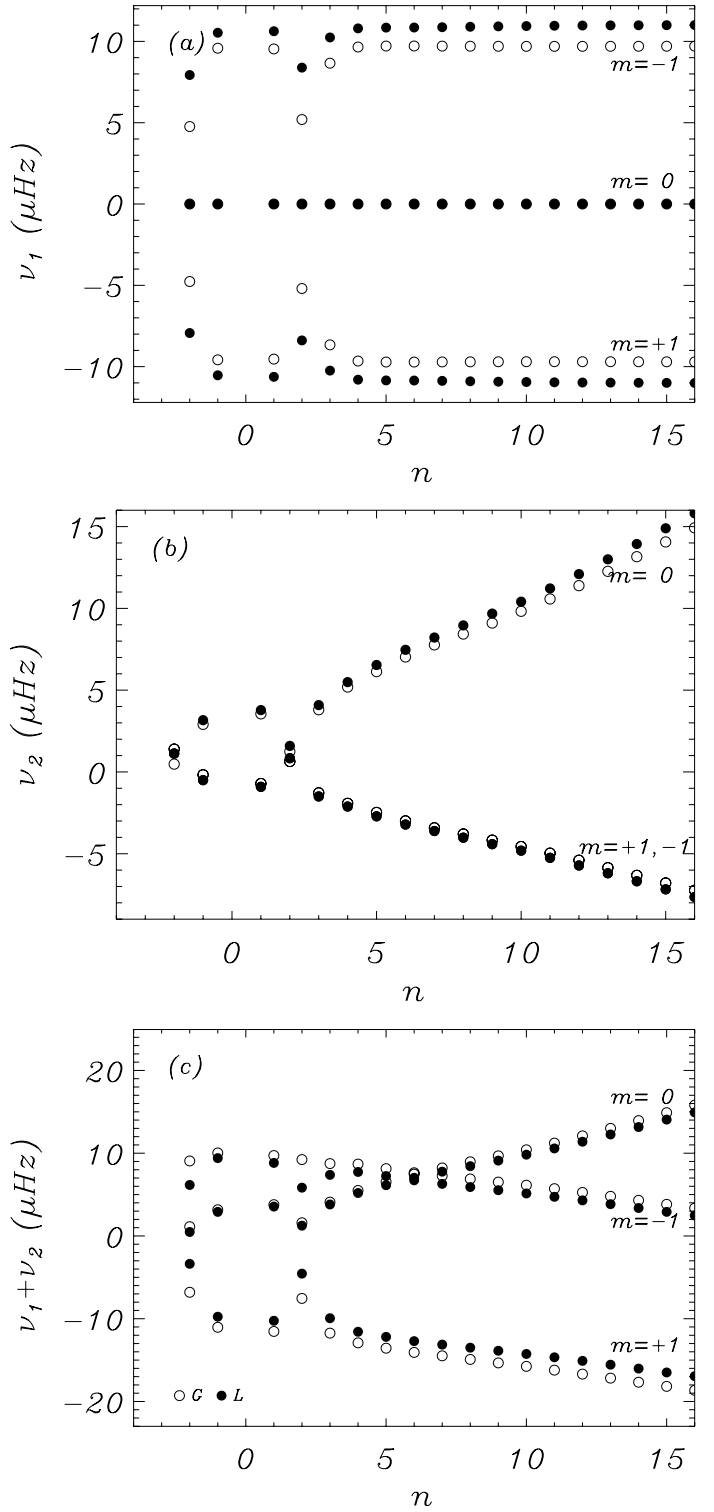

Fig. 4. First a) and second b) order frequency corrections $v=\omega / 2 \pi$ for $\ell=1$ modes as a function of the radial order $n$. Finally, both contributions $\omega_{1}+\omega_{2}$ are displayed in panel c). Empty and filled circles represent frequency corrections computed in the cases of uniform and shellular rotation, respectively.

through differences in their structural variables (pressure, density perturbations, etc.) rather than from differences in the wave propagation properties that appear as additional extra terms in the oscillation equations (see Appendix A).

\subsection{First-order frequency corrections}

In Fig. 4a, the first-order frequency corrections $\omega_{1}$ (Eq. (8)) are represented as a function of the radial order $n$. This figure can be seen seen as composed of two domains: a first one ( $n \leq$ 5) containing $g$ modes and mixed modes (hereafter $g p$ modes region) and a $p$-mode region $(n>5)$. As expected, modes in $g p$ region are more affected by shellular rotation than those in the $p$-mode region.
In Fig. 6, the frequency correction differences, $\Delta \omega_{1}=$ $\omega_{1}^{L}-\omega_{1}^{G}$, are represented as a function of their radial order $n$ for $\ell=1$ modes. These differences assess the effect of assuming a shellular rotation instead of uniform rotation. In the region of $g p$ modes, the effect of shellular rotation can reach up to $3 \mu \mathrm{Hz}$ for a few modes. For high radial order $n$, differences $\Delta \omega_{1}$, although smaller than for $g p$ modes, are significant and remain quite stable around $1 \mu \mathrm{Hz}$. These results can be explained by analyzing the contribution of shellular rotation to $\omega_{1}$ corrections (Eqs. (8), (10), and (12)), which arises from:

1) the effect of $\Omega(r)$ on the zeroth-order eigenfunctions $y_{01}$ and $z_{0}$ (corresponding to the radial and horizontal displacements, respectively) appearing in $C_{\mathrm{L}}$ and $J_{0}$ terms. Those eigenfunctions are obtained by solving the eigenvalue system, taking into account a shellular rotation, $\Omega(r)$, given in Appendix A (Eqs. (A.1)-(A.4)). The importance of this effect can be measured with the weighted function $f$ as defined by

$f(r)=\rho r^{4} y_{01}^{2}$,

where $y_{01}$ represents the radial displacement eigenfunction;

2) the explicit contribution of the rotation profile $\eta_{0}$ in $J_{0}$ (Eq. (12)). This latter term vanishes in the case of uniform rotation. For high-frequency $p$ modes, the horizontal component of the displacement is negligible $\left(\left|y_{01}\right| \gg z_{0}\right)$ and one has $J_{0} \sim\left\langle\eta_{0}\right\rangle$ where $\langle\cdot\rangle$ is a weighted average defined as:

$$
\langle\cdot\rangle \sim \frac{\int_{0}^{R} \rho r^{4} y_{01}^{2} \mathrm{~d} r}{\int_{0}^{R} \rho r^{4} y_{01}^{2} \mathrm{~d} r} .
$$

In Fig. 5, the weighted function $f(r)=\rho r^{4} y_{01}^{2}$ and $\eta_{0}(r) f(r)$ are then represented as functions of the radial distance $r$ for a high radial order $p$ mode (Fig. 5b) and for comparison in Fig. 5a for a mixed mode.

For $g p$ modes, the eigenfunctions (as shown by $f$ functions in Fig. 4a) present an inner maximum near the core $r / R \sim[0,0.2]$. As the kinetic energy of these modes are large in core-close regions, large differences between the uniform and the shellular rotation cases, i.e. in $\Delta \omega_{1}$, are reasonably expected.

For high radial-order $p$ modes, the implicit effects of shellular rotation on eigenfunctions $f_{\mathrm{L}}$ are represented by dashed lines and can be compared with $f_{G}$ for a uniform rotation. Maximum amplitudes of such modes are located near the stellar surface, and the eigenmodes do not differ much between the uniform and the shellular cases. The main contribution to the differences $\Delta \omega_{1}=1 \mu \mathrm{Hz}$ comes from the large increase in the rotation rate toward the center, which is accounted for by $\eta_{0}$ in $J_{0}$. Hence, negative values of $J_{0}$ explain that $\omega_{1}$ absolute values obtained in the case of a shellular rotation are lower than those obtained when a uniform rotation is assumed as seen in Fig. 4a.

\subsection{Second-order frequency corrections}

Second-order frequency corrections $\omega_{2}$ are found to be smaller than the first-order frequency corrections except at large 

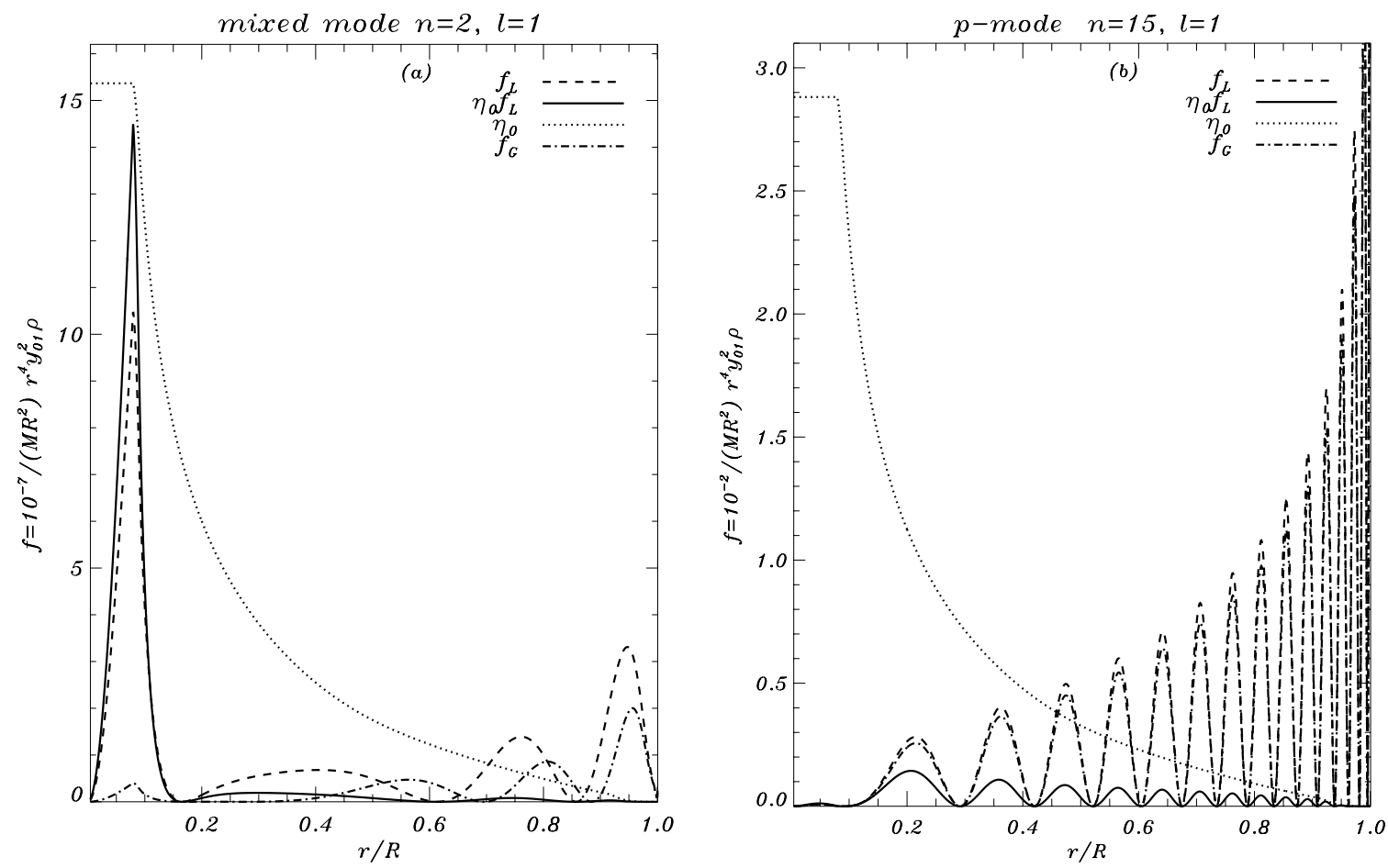

Fig. 5. Weighted $y_{01}$ functions versus the radial distance $r$ (normalized to the radius of the star $R$ ). Solid and dashed lines represent the $f$ functions computed for the $B_{\mathrm{L}}$ model. Dash-dotted lines represent the $f$ function (defined by Eq. (44)) computed for the $B_{G}$ model. Dotted lines represent the rotation profile given by the radial function $\eta_{0}(r)$ (scale adapted for clarity). Panel a) shows $f$ computed for the $n=2, \ell=1$ mixed mode, while panel b) represents $f$ computed for the $n=15, \ell=1 p$ mode. In both plots, the corresponding shellular rotation profile (same as Fig. 3) is also displayed.

frequency. They are presented in Fig. $4 \mathrm{~b}$ for $\ell=1$ modes, as well as the sums $\left(\omega_{1}+\omega_{2}\right)$ as a function of their radial order $n$. At low frequencies $(n \leq 5), \Delta \omega_{2}$ is comparable to the effect on second-order corrections $\left(\Delta \omega_{0}\right)$.

In the $p$-modes region, investigations of the individual terms in Eq. (9) reveal that $X_{2}$ and $Y_{2}$ are clearly dominant in that region. For given $\ell=1$ centroid modes, $\omega_{2}$ can be written as:

$\omega_{2}(\ell=1, m=0)=\frac{\bar{\Omega}^{2}}{\omega_{0}}\left[X_{1}^{\mathrm{T}}+X_{1}^{\mathrm{I}}+X_{2}\right]$.

In this expression, $X_{2}$ is found to be dominant mainly due to its $\omega_{0}$ dependence through the $\mathcal{I}_{\mathrm{c}}$ term (see Eq. (B.8)). Neglecting then $X_{1}^{\mathrm{T}}$ and $X_{1}^{\mathrm{I}}$ and considering $X_{2}(\ell=1, m=0)$, Eq. (46) reduces to:

$\omega_{2}(\ell=1, m=0) \sim \frac{2}{5} \frac{\bar{\Omega}^{2}}{\omega_{0}} \mathcal{J}_{\mathrm{c}}$.

For $m= \pm 1$ modes, $Y_{2}$ behaves like $X_{2}$ but with opposite sign. Similar to $X_{2}$, the presence of $C \omega_{0}^{2}$ in $\mathcal{J}_{\mathrm{c}}$ makes $Y_{2}$ dominant respect to $Y_{1}$. Thus the shape of the $m= \pm 1$ branches shown in Fig. $4 \mathrm{~b}$ can be explained by the competition between $X_{2}$ (positive) and $Y_{2}$ (negative), yielding:

$\omega_{2}(\ell=1, m \neq 0) \sim-\frac{1}{5} \frac{\bar{\Omega}^{2}}{\omega_{0}} \mathcal{J}_{\mathrm{c}}$.

More generally, for high radial order $(\ell \neq 0, m \neq 0)$ modes, one has (see also DG92):

$\omega_{2, m} \sim \frac{\left(\Lambda-3 m^{2}\right)}{4 \Lambda-3} \frac{\bar{\Omega}^{2}}{\omega_{0}} \mathcal{J}_{\mathrm{c}}$.
Unlike the first-order frequency corrections, the second-order $m \neq 0$ branches are not symmetric with respect to $m=0$ modes, and the asymmetry for a given $\ell$ can be written as:

$\omega_{2, m}+\omega_{2,-m}-2 \omega_{2,0}=-\frac{6 m^{2}}{4 \Lambda-3} \frac{\bar{\Omega}^{2}}{\omega_{0}} \mathcal{J}_{\mathrm{c}}$.

For high radial order $p$ modes, $\mathcal{J}_{\mathrm{c}}$ is given to a good approximation by

$\mathcal{I}_{\mathrm{c}} \sim \sigma_{0}^{2}\left\langle\mathcal{S}_{2}\right\rangle$,

where $\sigma_{0}^{2}=\omega_{0}^{2} /\left(G M / R^{3}\right)\left\langle\mathcal{S}_{2}\right\rangle$ is a weighted average of perturbations of structure (see Appendix B, Eq. (B.12)). Hence,

$\omega_{2}(\ell=1, m=0) \sim \frac{2}{5} \frac{\bar{\Omega}^{2}}{G M / R^{3}} \bar{\omega}_{0}\left\langle\mathcal{S}_{2}\right\rangle$,

and the increase of $\omega_{2}$ with $n$ clearly seen in Fig. $4 \mathrm{~b}$ arises from its $\omega_{0}$ dependence. In the $g p$-modes region, in contrast to pure $p$ modes, $X_{1}$ and $Y_{1}$ (more specifically $X_{1}^{\mathrm{T}}$ and $Y_{1}^{\mathrm{P}}$ ) dominate the structure rotational distortion effects given by $X_{2}$ and $Y_{2}$. In that case, both effects 1) and 2) mentioned above contribute in an intricate way.

The combination of both first- and second-order frequency corrections in Fig. 4 is then found to be dominated by the $\omega_{2}$ behavior at high frequency, and as was the case for first-order corrections, $\omega_{2}^{L}$ present absolute values higher than $\omega_{2}^{G}$. At low frequency ( $g p$-modes region), a complicated behavior results from both contributions $\left(\omega_{1}+\omega_{2}\right)$.

Figure 6 shows the frequency differences $\Delta \omega_{2}=\omega_{2}^{L}-\omega_{2}^{G}$. In general, $\Delta \omega_{2}<\Delta \omega_{1}$ over the whole range of considered 


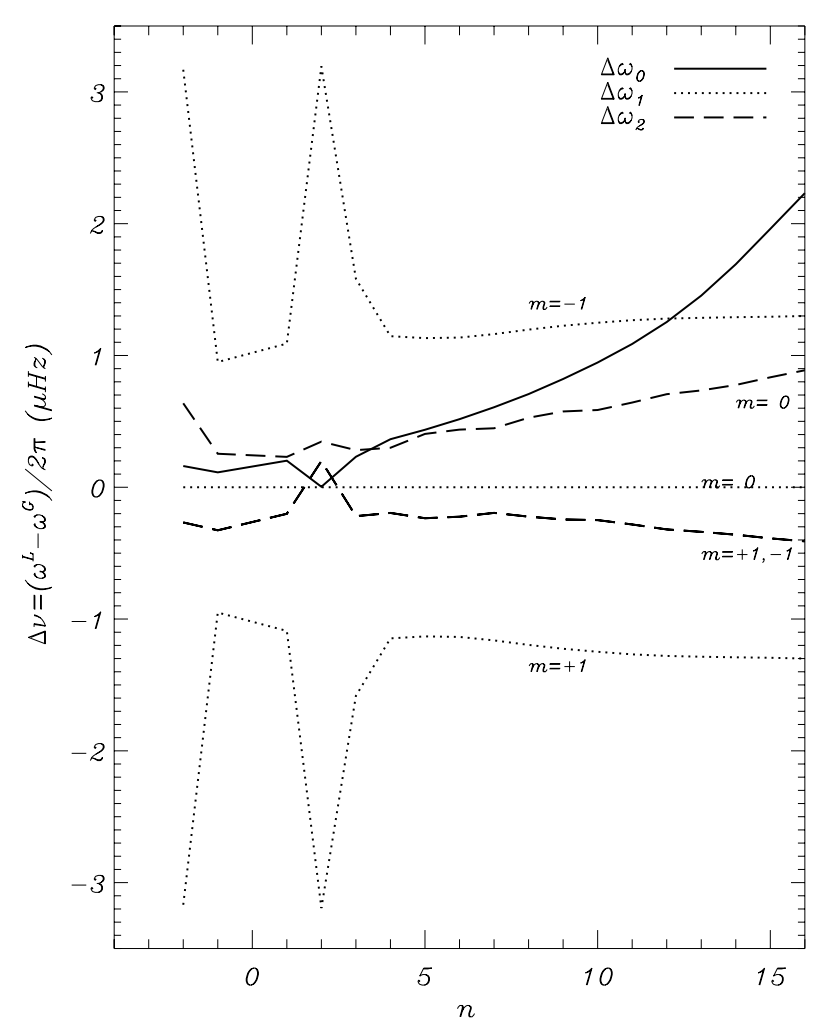

Fig. 6. Differences between oscillation frequencies $v=\omega / 2 \pi$ given in Fig. 4 as a function of their radial order $n$. Such differences are displayed for the zeroth-order $\Delta \omega_{0}=\omega_{0}^{L}-\omega_{0}^{G}$ (represented by a continuous line); the first-order $\Delta \omega_{1}=\omega_{1}^{L}-\omega_{1}^{G}$ indicated by dashed lines, and finally for second-order corrections $\Delta \omega_{2}=\omega_{2}^{L}-\omega_{2}^{G}$ represented by dotted lines.

radial orders. In contrast to the first-order results, the effect of shellular rotation on second-order frequency corrections $\left(\Delta \omega_{2}\right)$ is larger for $p$ modes than for $g p$ modes.

For high radial-order $p$ modes, the second-order frequency differences arise from:

$\Delta \omega_{2} \sim \frac{\bar{\Omega}^{2}}{G M / R^{3}}\left[\Delta\left(\omega_{0}\left\langle\mathcal{S}_{2}\right\rangle\right)\right]$,

with

$\Delta\left(\omega_{0}\left\langle\mathcal{S}_{2}\right\rangle\right)=\omega_{0}^{L}\left\langle\mathcal{S}_{2}\right\rangle^{L}-\omega_{0}^{G}\left\langle\mathcal{S}_{2}\right\rangle^{G}$.

As shown in Fig. 6, $\Delta \omega_{0}$ increases with the radial order $n$ in the $p$-mode region. The effect of this increase is lowered in $\Delta \omega_{2}$ by the change of $\left\langle\mathcal{S}_{2}\right\rangle$, which itself is mainly due to the derivatives of $\Omega(r)$ (i.e. $\left.b_{2}, \mathrm{~d} b_{2} / \mathrm{d} r\right)$.

For $g p$ modes, the difference in $\omega_{0}$ between both models is small (Fig. 6), and its contribution to $\Delta \omega_{2}$ (Eq. (44)) is less important. In this case, second-order effects of centrifugal and Coriolis forces on the stellar structure are dominant through terms in $\mathcal{J}_{\mathrm{c}}$ proportional to:

$r \frac{\mathrm{d} u_{2}}{\mathrm{~d} r}, r u_{2}, r b_{2}$

This is shown in equations describing the behavior of the pressure and density distributions in the presence of radial shellular rotation (Eqs. (74)-(80) in DG92). Moreover, second-order

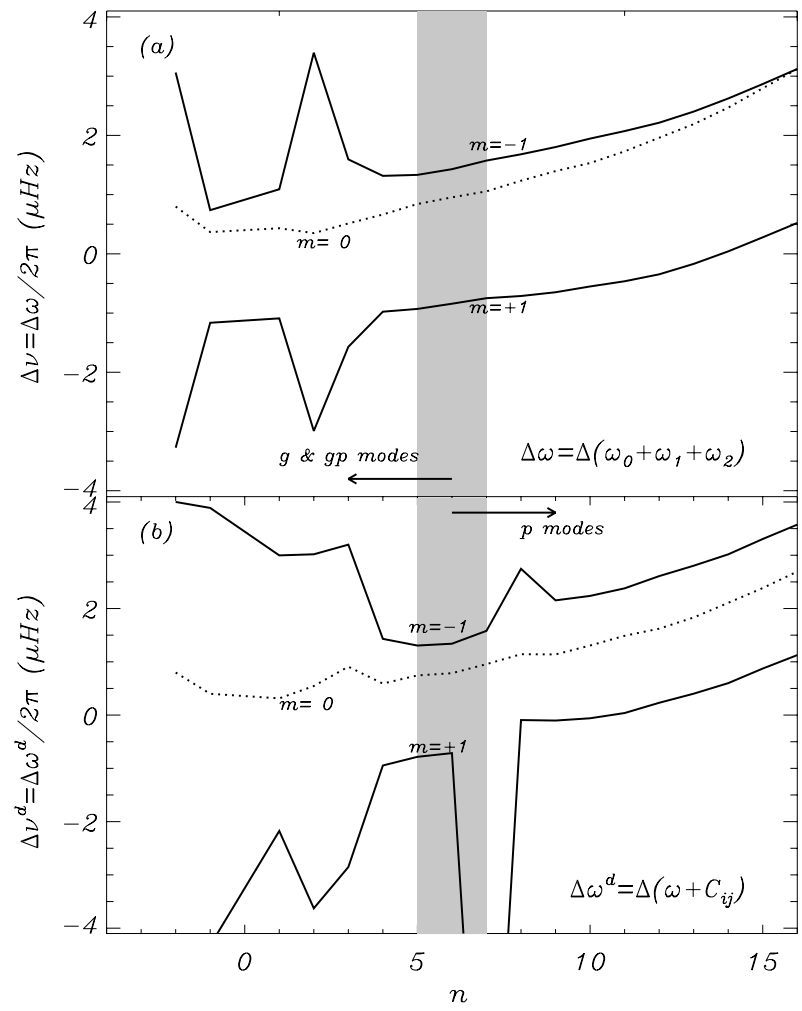

Fig. 7. a) Same as Fig. 6 but first- and second-order frequency corrections have been included. Symmetric solid-line branches represent from top to bottom, the differences for $m=-1$ and $m=+1$ mode frequencies respectively. For $m=0$ modes, differences are represented by a dotted line. Finally, panel b) is equivalent to a), but the the correction for near degeneracy is included. In both panels, the shaded region represents an indicative frontier between the region of $g$ and $g p$ modes (left side) and $p$ modes (right side).

effects of shellular rotation on the non-spherically symmetric component of the gravitational potential $\phi_{22}$ are found to be negligible, and $u_{2} \sim\left(1+\eta_{2}\right)$ in Eq. (B.8). Therefore, $\Delta \omega_{2}$ differences are dominated by the effect of $\Omega(r)\left(\eta_{0}\right)$ and of its derivatives $\left(b_{2}, \ldots\right)$. As was the case of first-order corrections, $\omega_{2}^{L}$ present absolute values that are higher than $\omega_{2}^{G}$.

\subsection{Full non-degenerate frequencies}

Finally, the total effect of differential rotation up to the second order on the frequency $\Delta \omega$ is displayed in Fig. 7a. The largest effects of shellular rotation on $g p$ modes (up to $\sim 3.4 \mu \mathrm{Hz}$ ) arise for $m= \pm 1$ modes and are dominated by $\Delta \omega_{1}$. In this region, the effects on zeroth order frequencies are much smaller. In contrast, for the $p$-modes region, $\Delta \omega$ are dominated by $\Delta \omega_{0}$ increasing with the radial order $n$ (see Sect. 5.1). Numerically, we find differences up to $0.5 \mu \mathrm{Hz}$ for $m=+1$ modes. For $m=-1$ modes, effects up to $3 \mu \mathrm{Hz}$ are seen to be of about the same order as those found for $m=0$ modes.

As already mentioned, the efficiency of the transport of angular momentum is modified by rotational mixing during the evolution of the star. The effect of shellular rotation on oscillation frequencies is expected to be reduced (in preparation) due to the smoothing of the variation of $\Omega / \Omega_{\mathrm{s}}$ at the edge of the 

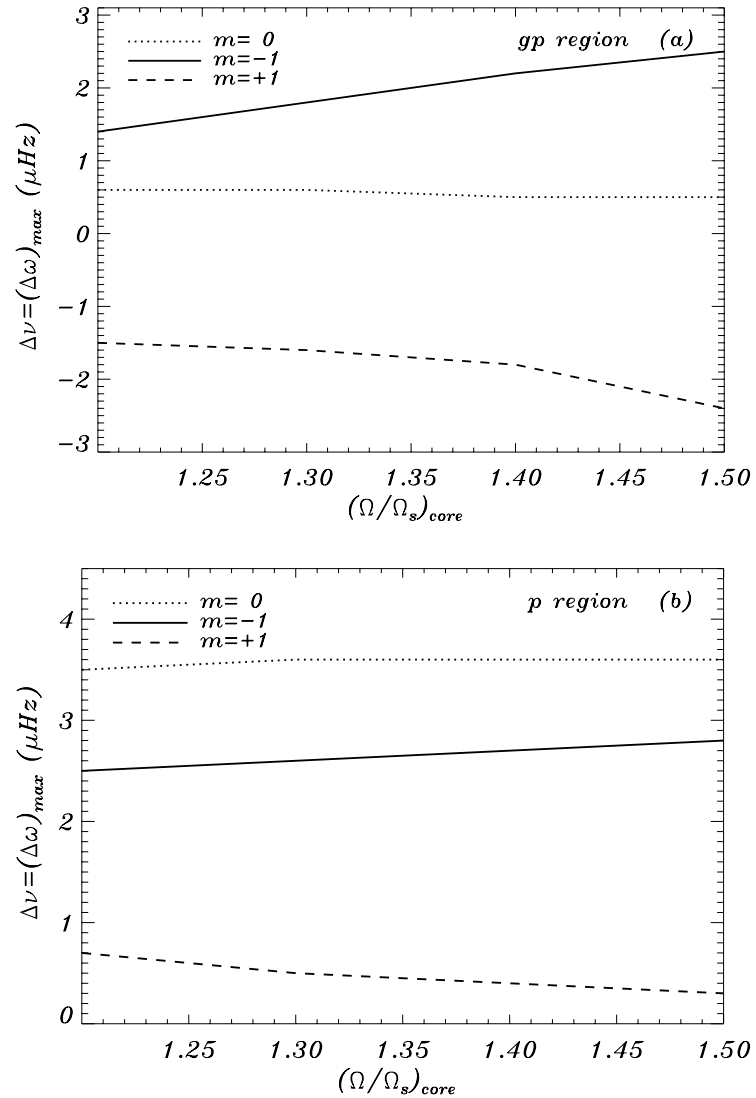

Fig. 8. Variation of $\Delta v=\Delta \omega / 2 \pi$ with $\Delta \omega=\Delta \omega_{0}+\Delta \omega_{1}+\Delta \omega_{2}$ as a function of the rotation rate of the uniformly rotating convective core normalized to the surface rotation rate $\Omega / \Omega_{\mathrm{s}}=1.2$. The effect of varying the rotation rate is shown for modes in $g p$ - and $p$-mode regions. The plots focus on $(\ell=1 ; m=0, \pm 1)$ modes for which $\Delta \omega$ gets a maximum value (see Fig. $7 \mathrm{a}$ ) $; n=3$ modes in the $g p$ domain (panel a)), and $n=16$ modes in the $p$-modes region (panel $\mathbf{b}$ ).

convective core (see Fig. 3). In order to simulate such a situation, theoretical oscillation spectra for the model $B_{L}$ were computed with core rotation rates in the range of $\Omega / \Omega_{\mathrm{s}}=[1.5,1.2]$. As expected, the major influence of such variations is found for $g p$ modes. The lower the rotation rate in the core, the lower the effect of shellular rotation in this region (see Fig. 8a). For the minimum rotation rate considered, $|\Delta \omega| \sim 1.4 \mu \mathrm{Hz}$ for $m=-1$ modes, and $|\Delta \omega| \sim 1.5 \mu \mathrm{Hz}$ for $m=+1$ modes is found. For $p$ modes (see Fig. 8b), differences remain close to those obtained for $\Omega / \Omega_{\mathrm{s}}=2.5$ (used throughout the present work).

\subsection{Correcting for near degeneracy}

Near degeneracy is taken into account as described in Sect. 3. The oscillation frequency of near-degenerate modes are computed according to Eqs. (18), (28), and (33) or Eqs. (18) and (37). In Fig. 7b, $\omega^{L}$ and $\omega^{G}$ are represented for $\left(\ell_{a}, \ell_{b}\right)=$ $(1,3)$ degenerate pairs. Near degeneracy is considered here for modes with $\left|\sigma_{a}-\sigma_{b}\right| \lesssim \Omega_{\mathrm{s}} /\left(G M / R^{3}\right)^{1 / 2} \sim 0.25$ (i.e. $\left|v_{a}-v_{b}\right| \sim 10 \mu \mathrm{Hz}$ here) corresponding to the surface rotation frequency of $B_{G}$ and $B_{L}$ models (see Table 1). As can be noticed for both models, modes in the $g p$ region are clearly the most af- fected by near degeneracy, with a few $m= \pm 1$ near-degenerate modes within the range of $n \lesssim 8$. This result was expected since such modes are probably closer to each other than are $p$ modes. In order to quantify how the oscillation frequencies are modified by near degeneracy, we define the coefficients $C_{a b}^{(1)}$ and $C_{a b}^{(2)}$ composed for first- and second-order contributions, where $a b$ subscripts represent the two modes affected by near degeneracy. According to the definitions in Eqs. (16)-(17), the "+" sign is for mode $a$ and the "-" sign is for mode $b$. In the case of near-degenerate modes with the same degree $\ell$, the firstand second-order coefficients are defined, respectively, as

$C_{a b}^{(1)} \equiv \tilde{\omega}_{1}-\omega_{1} \pm \frac{\Delta \omega_{0}}{2}, \quad C_{a b}^{(2)} \equiv \tilde{\omega}_{2}-\omega_{2}-\frac{\Delta \omega_{0}^{2}}{8 \bar{\omega}_{0}}$

In the case of two degenerate modes with different degrees $\ell_{a}=$ $\ell_{b} \pm 2$, the first-order coefficient $C_{a b}^{(1)}=0$ (see Sect. 3) and

$C_{a b}^{(2)} \equiv \tilde{\omega}_{2}-\omega_{2} \pm \frac{\Delta \omega_{0}}{2}$

The full expression for the adiabatic oscillation frequencies can be written as:

$\omega= \begin{cases}\omega_{0}+\left[\omega_{1}+C_{a b}^{(1)}\right]+\left[\omega_{2}+C_{a b}^{(2)}\right] & \text { if } \ell_{a}=\ell_{b} \\ \omega_{0}+\omega_{1}+\left[\omega_{2}+C_{a b}^{(2)}\right] & \text { if } \ell_{a}=\ell_{b} \pm 2 .\end{cases}$

In Fig. 9, for degenerate pairs $\left(\ell_{a}, \ell_{b}\right)=(0,2)$ (left panel) and for $\left(\ell_{a}, \ell_{b}\right)=(1,3)$ (right panel), the effect of near degeneracy is assessed by $C_{a b}^{(2)} / \omega_{0}$ as a function of the frequency for the cases of uniform and shellular rotation. As explained above, for such degenerate pairs, only the second-order degenerate coefficient is nonnull. It can be shown that the dominant terms correspond to $\omega_{2, a b}^{\mathrm{D}}$ and $\omega_{2, a b}^{\mathrm{T}}$ (Eqs. (C.14) and (C.11)). The first one takes into account the distortion of the structure of the star through the structure quantities $d_{1}$ and its derivative $d_{2}$ (Eq. (C20)). The second one, $\omega_{2, a b}^{\mathrm{T}}$ corresponds to the toroidal component of eigenfunctions. For the range of frequencies treated here, the global effect remains small for high-frequency $p$ modes with respect to what is found in the $g p$-modes region, where $\omega_{2, a b}^{\mathrm{D}}$ and $\omega_{2, a b}^{\mathrm{T}}$ are of the same order. This explains that differences between differential and uniform rotation are mainly found for gp modes. Such variations can represent a few percent of the value of frequencies not corrected by near degeneracy. For $m=0$ modes, compensations of dominant terms turn out to yield a smaller effect of near degeneracy for $m=0$ modes seen in Fig. 9 (left panel), compared to those with $m \neq 0$ (right panel). For modes in the high frequency $p$-mode region, the near degeneracy effects shown in Fig. 9 can be studied in the asymptotic approximation by analyzing individual terms of Eq. (C.14) in Appendix C.3. This analysis reveals that for large frequency $p$ modes the small separation corresponding to $v_{n-1, \ell}-v_{n, \ell+2}$ is small enough with respect to the rotation rate of the star to degenerate all $(\ell, \ell+2)$ pairs. In this context (asymptotic regime), near degeneracy contributes with a slowly-increasing term with radial order, dominated by $\omega_{2, a b}^{\mathrm{D}}$ (see details in Sect. C.3.1), which is given to a good approximation by

$\omega_{2, a b}^{\mathrm{D}} \sim \frac{\bar{\Omega}^{2}}{G M / R^{3}} \frac{24}{7 \sqrt{5}} \bar{\omega}_{0}\left\langle\mathcal{S}_{2}\right\rangle$, 

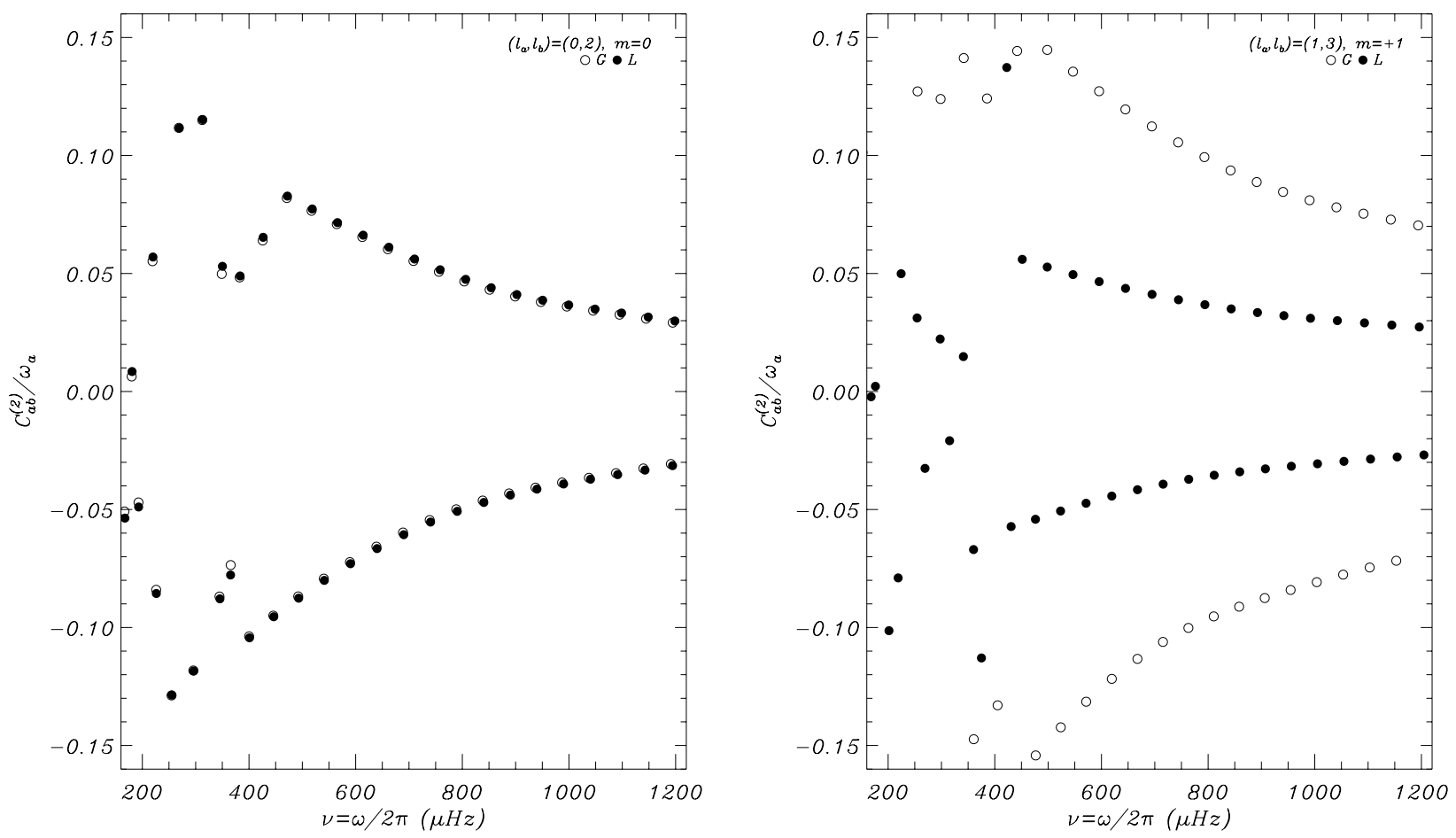

Fig. 9. Effect of the second-order near-degenerate coefficients $C_{a b}^{(2)} / \omega_{a}$ and $C_{a b}^{(2)} / \omega_{b}$ as a function of the frequency $(v=\omega / 2 \pi)$ for degenerate pairs $\left(\ell_{1}, \ell_{2}\right)=(0,2)$ (left panel) and $\left(\ell_{1}, \ell_{2}\right)=(1,3)$ (right panel). Empty and filled circles represent degenerate coefficients in the cases of uniform and shellular rotation, respectively.

for $\left(\ell_{a}, \ell_{b}\right)=(0,2)$ degenerate pairs and

$\omega_{2, a b}^{\mathrm{D}} \sim \frac{\bar{\Omega}^{2}}{G M / R^{3}} \frac{12}{5} \sqrt{\frac{2}{7}} \bar{\omega}_{0}\left\langle\mathcal{S}_{2}\right\rangle$,

for $\left(\ell_{a}, \ell_{b}\right)=(1,3)$ degenerate pairs. For high-frequency $p$ modes, $C_{a b}^{(2)}$ increases as $\bar{\omega}_{0}$, so the ratio $C_{a b}^{(2)} / \bar{\omega}_{0}$ remains nearly constant. Figure 9 also reveals that the behavior of the $C_{a b}^{(2)}$ coefficients differs when differential or uniform rotation is considered. These differences are found to be significant for $m \neq 0$ degenerate modes and are mainly caused by differences at zeroth order ( $\omega_{0}$ in Eqs. (58) and (59)).

For modes with $\Delta \ell=0$ (for which $C_{a b}^{(1)} \neq 0$ ), further information can be obtained through the second-to-first order coefficient ratio

$q_{(2,1)}=\left|\frac{C_{a b}^{(2)}}{C_{a b}^{(1)}}\right|$.

In the present case, such degenerate pairs (which represent only a few marginal cases) are found for $\ell=2$ and $\ell=3$, for which $C_{a b}^{(2)} \sim C_{a b}^{(1)}$. When considering differential rotation, mixed modes $(n=-6 \ldots 2)$ present slightly higher (from $1 \%$ to $20 \%$, in absolute value) second-order degenerate coefficients than first order ones. Although the correction for near degeneracy is thus less important in the case of shellular rotation, it cannot be neglected.

\section{Discussion and conclusions}

In the present work, adiabatic oscillation frequencies of stellar models with intermediate mass have been computed, which include the effects of rotation. Following the perturbation formalism of DG92 and SGD98, we built a numerical code that provides adiabatic eigenfrequencies corrected up to the second order in the rotation rate $\Omega$ allowing a radial dependence of $\Omega=\Omega(r)$. Two particular cases for the transport of the angular momentum in stellar interiors are investigated: on the one hand, an instantaneous transport of the angular momentum thus leading to a uniform rotation profile. On the other hand, a shellular rotation profile $\Omega(r)$ is assumed and derived from the assumption of local conservation of angular momentum. Our study was focused on $1.80 M_{\odot}$ equilibrium models of typical $\delta$ Scuti stars with a surface rotation of $100 \mathrm{~km} \mathrm{~s}^{-1}$.

As mentioned in the introduction, the seismology of A-F rapidly rotating stars faces many difficulties, among which the most severe is probably mode identification (see reviews Goupil et al. 2005; and Dupret et al. 2005, for $\delta$ Scuti star and $\gamma$ Dor stars, respectively). We stress that the present study does not intend to solve the problem of mode identification, which now begins to be handled with confidence using various techniques (see Handler 2005, and references therein). In particular, diagnostics based on multicolor photometry (Garrido et al. 1990) are employed nowadays for mode identification. In the framework of the forthcoming space mission Corot, the color photometric data from the exo-planet camera will provide very useful information for this task. In any case, whether mode identification is successfully performed or not, calculation of models and associated oscillation frequencies as close to reality as possible is necessary for a one-to-one frequency comparison between observed and computed frequencies (i.e. spectrum of frequency differences or frequency histograms). 
At that level, it is important to be able to compute frequencies with different physical assumptions and assess their respective effects for establishing possible seismic diagnostics. The present study was done along these lines.

To estimate the numerical and theoretical uncertainties of the computed eigenfrequencies, as well as the validity of the adopted perturbation technique is not an easy task. Here we have performed a one-to-one eigenfrequency comparison between eigenfrequencies computed with the present numerical code and those computed with the Warsaw oscillation code restricted to second-order perturbation frequency corrections. This exercise also implies comparison of the different perturbation techniques employed in each code. The input model for both codes is the same, $B_{G}$, with uniform rotation (Table 1). The results show that differences less than $0.15 \mu \mathrm{Hz}$ are obtained most often and are never larger than $0.5 \mu \mathrm{Hz}$. Both oscillation codes are different in their numerical schema, treatments of boundary conditions, and other technical computation characteristics. The adopted perturbation technique is also different since in one case, the first-order frequency correction is explicitly calculated (present code), whereas in the other case, the first-order effect of rotation is implicitly included in the eigensystem. The results of the comparison thus give some estimate of the precision of the frequencies computed here and give us some confidence in their calculation.

Note also that triple-mode interaction occurs between three modes such as $\ell_{a}=\ell_{b}=\ell_{c}-2$ or $\ell_{a}=\ell-b-2=\ell_{c}-2$ in the adopted perturbation formalism here, although only occasionally. These triple-mode interactions correspond to a doublemode interaction in the SGD98formalism since in this last one, the case $l_{a}=l_{b}$ near degeneracy is implicitly included.

Big effects - up to $3 \mu \mathrm{Hz}$ - of shellular rotation compared with a uniform rotation are observed in the low frequency region where $g p$ modes are encountered. This is expected, as the rotation profile varies rapidly in the inner layers at the edge of the convective core where $g p$ modes have large amplitudes.

In the case of higher frequency $p$ modes, frequency differences up to $3 \mu \mathrm{Hz}$ are predicted between a uniform and a shellular rotation. This is due to the structure deformation caused by the centrifugal force, which mainly affects the zeroth-order oscillation frequencies.

It is found that the impact of reducing artificially the core rotation rates of models by $50 \%$ principally affects those $g p$ modes for which the effects shellular rotation are drastically reduced to $0.6-1 \mu \mathrm{Hz}$. In addition, the frequency changes due to a shellular rotation compared to a uniform rotation are found to be much larger for these intermediate rotators than are third order $\left(\Omega^{3}\right)$ effects (around $0.04 \mu \mathrm{Hz}$ for the region of $g$ and gp modes, and around $0.02 \mu \mathrm{Hz}$ for $p$ modes, for a $100 \mathrm{~km} \mathrm{~s}^{-1}$ star model). This represents around $3.3 \%$ and $2 \%$ of effects found for the core-reduced rotation models discussed above.

Likewise, note that such effects can be even larger for different kinds of stars (Suárez et al., in preparation). Observational evidence for this have already been obtained for the early-B type stars HD 129929 and HD 29248 (Aerts et al. 2003; and Pamyatnykh et al. 2004, respectively).

For the models and rotation rates investigated here, quite a number of modes are near degenerate, and a double-mode interaction had to be computed for these modes. On the other hand, we find that only a few modes require a triple-mode interaction $\ell=0,2,4$ or $\ell=1,3,5$ : one set at high frequency $n=9,10,11$ and two or three sets at low frequency (in the fundamental mode vicinity). For these triple near-degenerate modes, we encounter all possible cases of the two closest modes among the three being either $\ell=0, \ell=2$ or $\ell=0, \ell=4$ or $\ell=2,4$; this is the same for $\ell=1,3,5$ modes. The effect of taking triple interaction into account instead of double interaction can be quite different depending on the configuration of the 3 modes (closeness of the frequencies). We find that the effect can occasionally amount up to a few $\mu \mathrm{Hz}$.

In view of our different tests, we conclude that if one looks for seismic signatures of shellular rotation that are smaller than $0.5 \mu \mathrm{Hz}$, we have to resort to the SGD98 and Karami et al. (2005) formalism and include triple-mode interaction whenever it is necessary.

For higher rotation rates, perturbation techniques are no longer useful, even if still valid, when the effects of rotation are large enough that triple-mode interaction must be systematically taken into account for most modes; this situation indeed would suggest that interaction that is higher than triple-mode ought to be included. Then non-perturbative techniques, such as the one developed by Lignières \& Rieutord (2004) and Rees et al. (2005, in prep.), will have to be used.

In the framework of the forthcoming space mission COROT (Baglin \& The COROT Team 1998), precisions of $0.1 \mu \mathrm{Hz}$ on mode detection, and around $0.5 \mu \mathrm{Hz}$ on splitting resolutions, are expected to be observed. With such accuracy, the effects of shellular rotation are likely to detectable, provided numerical eigenfrequencies reach this level of precision. Finally, we stress that it is possible to remove contaminations of the high-order (second and higher) effects of rotation from the rotational splitting in order to recover the true rotation profile with the usual inversion techniques: this has been outlined by (Dziembowski \& Goupil 1998; Goupil et al. 2004) and will be described in more detailed in a forthcoming paper. At low rotation rates, this task will become even easier, since in this case, the effect of differential rotation on oscillation frequencies is dominated by structure terms in zeroth and first-order frequency corrections.

Acknowledgements. This study would not have been possible without the financial support of the European Marie Curie action MERGCT-2004-513610. It was also financed partially by the Spanish "Consejería de Innovación, Ciencia y Empresa" from the "Junta de Andalucía" local government, and by the Spanish Plan Nacional del Espacio under project ESP2004-03855-C03-01. We gratefully thank W. Dziembowski for his pertinent critical remarks.

\section{References}

Aerts, C., Thoul, A., Daszyńska, J., et al. 2003, Science, 300, 1926 Baglin, A., \& The COROT Team. 1998, in New Eyes to See Inside the Sun and Stars, IAU Symp., 185, 301

Breger, M. 2000, in Delta Scuti and Related Wtars, Reference Handbook and Proceedings of the 6th Vienna Workshop in Astrophysics, held in Vienna, Austria, 4-7 August, 1999, ed. M. Breger, \& M. Montgomery (San Francisco: ASP), ASP Conf. Ser., 210, 3 
Christensen-Dalsgaard, J. 1998, Lecture notes on Stellar Oscillations, quatrième édition edn., Institut for Fysikk og Astronomi, Université de Aarhus

Daszyńska-Daszkiewicz, J., Dziembowski, W. A., Pamyatnykh, A. A., \& Goupil, M.-J. 2002, A\&A, 392, 151

Daszyńska-Daszkiewicz, J., Dziembowski, W. A., \& Pamyatnykh, A. A. 2003, Ap\&SS, 284, 133

Denissenkov, P. A., \& Tout, C. A. 2000, MNRAS, 316, 395

Dupret, M. A., Grigahcène, A., De Ridder, J., et al. 2005, in Mem. S. A. It. Stellar Pulsation and Evolution, Rome 2005, in press

Dziembowski, W. A., \& Goode, P. R. 1992, ApJ, 394, 670

Dziembowski, W. A., \& Goupil, M.-J. 1998, in The First MONS Workshop: Science with a Small Space Telescope, held in Aarhus, Denmark, June 29-30, 1998, ed. H. Kjeldsen, \& T. R. Bedding, Aarhus Universitet, 69

Garrido, R., Garcia-Lobo, E., \& Rodriguez, E. 1990, A\&A, 234, 262

Goupil, M. J., \& Talon, S. 2002, in Radial and Nonradial Pulsationsn as Probes of Stellar Physics, IAU Coll., 185, ASP Conf. Ser., 259, 306

Goupil, M.-J., Dziembowski, W. A., Pamyatnykh, A. A., \& Talon, S. 2000, in Delta Scuti and Related Wtars, Reference Handbook and Proceedings of the 6th Vienna Workshop in Astrophysics, held in Vienna, Austria, 4-7 August, 1999, ed. M. Breger, \& M. Montgomery (San Francisco: ASP), ASP Conf. Ser., 210, 267

Goupil, M. J., Samadi, R., Lochard, J., Dziembowski, W. A., \& Pamyatnykh, A. 2004, in Stellar Structure and Habitable Planet Finding, ESA SP-538, 133

Goupil, M. J., Dupret, M. A., Samadi, R., et al. 2005, J. Astrophys. Astr., 26, 249

Handler, G. 2000, in The Impact of Large-Scale Surveys on Pulsating Star Research, IAU Coll., 176, ASP Conf. Ser., 203, 408
Handler, G. 2005, J. Astrophys. Astr., 26, 241

Heger, A., Langer, N., \& Woosley, S. E. 2000, ApJ, 528, 368

Karami, K., Christensen-Dalsgaard, J., Pijpers, F. P., Goupil, M., \& Dziembowski, W. A. 2005, ArXiv Astrophysics e-prints

Kippenhahn, R., \& Weigert, A. 1990, Stellar structure and evolution, Astronomy and Astrophysics library (Springer-Verlag)

Lignières, F., \& Rieutord, M. 2004, in IAU Symp., 414

Maeder, A., \& Meynet, G. 2000, ARA\&A, 38, 143

Michel, E., Chevreton, M., Belmonte, J. A., et al. 2000, in The Impact of Large-Scale Surveys on Pulsating Star Research, IAU Coll., 176, ASP Conf. Ser., 203, 483

Palacios, A., Talon, S., Charbonnel, C., \& Forestini, M. 2003, A\&A, 399, 603

Pamyatnykh, A. A. 2003, Ap\&SS, 284, 97

Pamyatnykh, A. A., Handler, G., \& Dziembowski, W. A. 2004, MNRAS, 350, 1022

Saio, H. 1981, ApJ, 244, 299

Soufi, F., Goupil, M. J., \& Dziembowski, W. A. 1998, A\&A, 334, 911

Soufi, F., Goupil, M. J., Dziembowski, W. A., \& Sienkiewicz, H. 1995, in Astrophysical Applications of Stellar Pulsation, IAU Coll., 155, ASP Conf. Ser., 83, 321

Suárez, J. C. 2002, Ph.D. Thesis

Talon, S., Zahn, J.-P., Maeder, A., \& Meynet, G. 1997, A\&A, 322, 209

Templeton, M. R., McNamara, B. J., Guzik, J. A., et al. 1997, AJ, 114, 1592

Unno, W., Osaki, Y., Ando, H., Saio, H., \& Shibahashi, H. 1989, Nonradial oscillations of stars (Nonradial oscillations of stars, Tokyo: University of Tokyo Press), 2nd edn.

Zahn, J.-P. 1992, A\&A, 265, 115 


\section{Online Material}




\section{Appendix A: Oscillation frequencies of a pseudo-rotating model}

Considering an effective gravity, $g_{\text {eff }}$ (Eq. (39)), and a rotation profile, $\Omega(r)$ (Eq. (7)), the following dimensionless quantities are defined as in SGD98:

$$
\begin{aligned}
& y_{01}=\frac{\xi_{r}}{r} \\
& y_{02}=\frac{1}{g_{\mathrm{eff}} r}\left(\phi^{\prime}+\frac{p^{\prime}}{\rho}\right) \\
& y_{03}=\frac{\phi^{\prime}}{g_{\mathrm{eff}} r} \\
& y_{04}=\frac{1}{g_{\mathrm{eff}}} \frac{\mathrm{d} \phi \prime}{\mathrm{d} r} .
\end{aligned}
$$

Following Unno et al. (1989), we obtain a linearized eigenvalue system:

$x \frac{\mathrm{d} y_{01}}{\mathrm{~d} x}=\lambda-3 y_{01}+\frac{\Lambda}{C_{r} \sigma_{0}^{2}} y_{02}$

$x \frac{\mathrm{d} y_{02}}{\mathrm{~d} x}=\left(C_{r} \sigma_{0}^{2}-A^{*}\right) y_{01}+\left(A^{*}+1-U_{\chi}\right) y_{02}-A^{*} y_{03}$

$x \frac{\mathrm{d} y_{03}}{\mathrm{~d} x}=\left(1-U_{\chi}\right) y_{03}+y_{04}$

$x \frac{\mathrm{d} y_{04}}{\mathrm{~d} x}=\frac{U}{1-\sigma_{r}}\left[A^{*} y_{01}+V_{g}\left(y_{02}-y_{03}\right)\right]+\Lambda y_{03}-U_{\chi} y_{04}$,

with $x=r / R$ and $R$ the stellar radius, where the following classical notations from Unno et al. (1989) are used

$A^{*}=\frac{1}{\Gamma} \frac{\mathrm{d} \ln p}{\mathrm{~d} \ln r}-\frac{\mathrm{d} \ln \rho}{\mathrm{d} \ln r}$

$V=-\frac{\mathrm{d} \ln p}{\mathrm{~d} \ln r}, \quad V_{g}=\frac{V}{\Gamma_{1}}$

$U=\frac{\mathrm{d} \ln M_{r}}{\mathrm{~d} \ln r}$

$\Lambda=\ell(\ell+1)$,

and with $U_{\chi}$ defined as follows:

$U_{\chi}=U+\chi$.

We also define, as in SGD98, the following variables

$$
\begin{aligned}
C & =\left(\frac{r}{R}\right)^{3} \frac{M}{M_{r}}, \quad C_{r}=\frac{C}{1-\sigma_{r}} \\
\sigma_{0}^{2} & =\frac{\omega_{0}^{2} R^{3}}{G M} ; \quad \sigma_{r}=\frac{\mathcal{A}_{\mathrm{c}}}{g} \\
\chi & =\frac{\mathcal{A}_{\mathrm{c}}}{g_{\mathrm{eff}}}\left(U-3+\frac{\mathrm{d} \Omega^{2} / \bar{\Omega}^{2}}{\mathrm{~d} r}\right) \\
\lambda & =V_{g}\left(y_{01}-y_{02}+y_{03}\right),
\end{aligned}
$$

where $\mathcal{A}_{\mathrm{c}}$ is the radial component of the centrifugal acceleration defined in Eq. (41); $M, m_{r}$ respectively are the stellar mass and the mass enclosed in the sphere of radius $r$. This system is solved with the following boundary conditions:

$$
\begin{aligned}
& y_{02}+y_{01} \frac{3}{V_{g}}=0,3 y_{01}+y_{04}=0 \quad(\ell=0) \\
& y_{01}-y_{02} \frac{\ell}{C_{r} \sigma_{0}^{2}}=0, y_{04}-\ell y_{03}=0 \quad(\ell \neq 0)
\end{aligned}
$$

at the center of the star and,

$$
\begin{aligned}
& y_{01}=1 \\
& y_{04}+(\ell+1) y_{03}=0 \\
& y_{01}\left(1+\frac{\Lambda}{V C \sigma_{0}^{2}}-\frac{4+C \sigma_{0}^{2}}{V}\right)-y_{02}\left(1-\frac{\Lambda}{V C \sigma_{0}^{2}}\right) \\
& +y_{03}\left(1+\frac{\ell+1}{V}\right)=0
\end{aligned}
$$

at the stellar surface. The resulting eigenvalues correspond to the oscillation frequencies of a pseudo-rotating model (with their associated eigenfunctions).

\section{A.1. First-order perturbed eigenfuctions}

Considering dimensionless variables equivalent to Eqs. (A.1)-(A.4) with first-order perturbed quantities $\left(\xi_{1, r}, \phi_{1}^{\prime}\right.$, $\left.p_{1}^{\prime}\right)$, and the zeroth-order solutions obtained from Eq. (A.5), first-order perturbed eigenfuctions can be calculated from the following system:

$$
\begin{aligned}
x \frac{\mathrm{d} y_{1}}{\mathrm{~d} x}= & \lambda_{1}-3 y_{1}+\frac{\Lambda}{C_{r} \sigma_{0}^{2}} y_{2} \\
& +\left(y_{01}+z_{0}\right)\left(1+\eta_{0}\right)-\left(\eta_{0}+\sigma_{1}\right) \Lambda z_{0} \\
x \frac{\mathrm{d} y_{2}}{\mathrm{~d} x}= & \left(C_{r} \sigma_{0}^{2}-A^{*}\right) y_{1}+\left(A^{*}+1-U_{\chi}\right) y_{2}-A^{*} y_{3} \\
& +\left(\sigma_{1}+\eta_{0}\right) y_{01}-\left(1+\eta_{0}\right) z_{0} \\
x \frac{\mathrm{d} y_{3}}{\mathrm{~d} x}= & \left(1-U_{\chi}\right) y_{3}+y_{4} \\
x \frac{\mathrm{d} y_{4}}{\mathrm{~d} x}= & \frac{U}{1-\sigma_{r}}\left[A^{*} y_{1}+V_{g} y_{2}-V_{g} y_{3}\right]+\Lambda y_{3}-U_{\chi} y_{4}
\end{aligned}
$$

where $\lambda_{1}=V_{g}\left(y_{1}-y_{2}+y_{3}\right)$ and

$z_{0}=\frac{y_{02}}{C \sigma_{0}^{2}}$

The horizontal component of $\boldsymbol{\xi}_{1}$ can be written as follows:

$z_{1}=\frac{y_{2}}{C \sigma_{0}^{2}}+\frac{1+\eta_{0}}{\Lambda} y_{01}+\left(\frac{1+\eta_{0}}{\Lambda}-\sigma_{1}\right) z_{0}$,

where $\sigma_{1}=C_{\mathrm{L}}-J_{0}$ represents the first-order correction of the corresponding eigenfrequency.

\section{Appendix B: $X_{i}$ and $Y_{i}$ expressions in Saio's notation}

The terms $X_{i}$ and $Y_{i}$ are constructed from $\omega_{2}^{\mathrm{I}}, \omega_{2}^{\mathrm{T}}, \omega_{2}^{\mathrm{P}}$ and, $\omega_{2}^{\mathrm{D}}$ (see Sect. 2). It is convenient to split $X_{1}$ and $Y_{1}$ as follows:

$X_{1}=X_{1}^{I}+X_{1}^{T}$

$Y_{1}=Y_{1}^{I}+Y_{1}^{T}+Y_{1}^{P}+Y_{1}^{0}$.

These two terms include both, the toroidal component of the first order perturbation of the displacement eigenfunction and an effect of inertia. The different components of $X_{1}$ are given by the following analytic expressions:

$$
X_{1}^{I}=\frac{1}{(8 \Lambda-6) I_{0}} \int_{0}^{R} b_{2}\left[2(\Lambda-1) y_{01}^{2}-\Lambda y_{01} z_{0}\right] \rho_{0} r^{4} \mathrm{~d} r
$$


J. C. Suárez et al.: Effects of shellular rotation on adiabatic oscillations, Online Material p 3

$$
\begin{aligned}
X_{1}^{T}= & \frac{2}{I_{0}} \int_{0}^{R}\left(1+\eta_{0}\right)^{2}\left[\left(y_{01}-\ell z_{0}\right)^{2} \frac{(\ell+1)(\ell+2)}{4(\ell+1)^{2}-1}\right. \\
& \left.+\left(y_{01}+(\ell+1) z_{0}\right)^{2} \frac{\ell(\ell-1)}{4 \ell^{2}-1}\right] \rho_{0} r^{4} \mathrm{~d} r .
\end{aligned}
$$

Respectively, the components of $Y_{1}$ are expressed as:

$$
\begin{aligned}
Y_{1}^{I}= & C_{l}-\frac{1}{2} \\
& -\frac{1}{2 I_{0}} \int_{0}^{R} \eta_{2}\left[y_{01}^{2}+(\Lambda-2) z_{0}^{2}-4 y_{01} z_{0}\right] \rho_{0} r^{4} \mathrm{~d} r \\
Y_{1}^{T}= & -\frac{2}{I_{0}} \int_{0}^{R}\left(1+\eta_{0}\right)^{2}\left[\left(y_{01}-\ell z_{0}\right)^{2} \frac{\ell+2}{\ell+1} \frac{1}{4(\ell+1)^{2}-1}\right. \\
& \left.+\left(y_{01}+(\ell+1) z_{0}\right)^{2} \frac{\ell-1}{\ell} \frac{1}{4 \ell^{2}-1}\right] \rho_{0} r^{4} \mathrm{~d} r
\end{aligned}
$$$$
Y_{1}^{P}=\frac{2}{I_{0}} \int_{0}^{R}\left(1+\eta_{0}\right)\left[z_{0} y_{1}+z_{1} y_{01}-y_{01} y_{1}\right.
$$$$
\left.-(\Lambda-1) z_{1} z_{0}\right] \rho_{0} r^{4} \mathrm{~d} r
$$

$Y_{1}^{0}=\frac{1}{2}\left(\sigma_{1}-1\right)^{2}$

Furthermore, $\mathrm{X}_{2}$ et $Y_{2}$ contain the effect of deformation of the star due to the non-spherical component of the centrifugal acceleration. They are given by:

$X_{2}=\frac{\Lambda}{4 \Lambda-3} \mathcal{I}_{\mathrm{c}} \quad Y_{2}=\frac{-3}{4 \Lambda-3} \mathcal{I}_{\mathrm{c}}$

with

$$
\begin{aligned}
\mathcal{I}_{\mathrm{c}}= & \frac{1}{2 I_{0}} \int_{0}^{R}\left\{C \sigma _ { 0 } ^ { 2 } \left[\left(2 y_{01}^{2}+3 z_{0}^{2}\right)\left(r \frac{\mathrm{d} u_{2}}{\mathrm{~d} r}+(4-U) u_{2}\right)\right.\right. \\
& \left.+2 z_{0}^{2} \Lambda u_{2}+z_{0} y_{01}\left(\Psi_{1}+6 u_{2}\right)\right] \\
& +y_{01}\left(\lambda-y_{01}\left(A^{*}+V_{g}\right)\right) U u_{2}-y_{01} y_{03}\left(\Psi_{1}+2 \Lambda u_{2}\right) \\
& -y_{01} y_{04}\left(3 r \frac{\mathrm{d} u_{2}}{\mathrm{~d} r}+u_{2}(10-3 U)\right) \\
& -2 z_{0} y_{04} \Lambda u_{2}-\left[y_{01}^{2}\left(C \sigma_{0}^{2}+3-U\right)+z_{0}^{2} C \sigma_{0}^{2}(\Lambda-3)\right. \\
& \left.+3 z_{0} y_{01}-2 y_{01} y_{04}-2 z_{0} y_{03}(\Lambda-3)\right] b_{2} \\
& \left.-y_{01}{ }^{2} r \frac{\mathrm{d} b_{2}}{\mathrm{~d} r}\right\} \rho_{0} r^{4} \mathrm{~d} r
\end{aligned}
$$

with $u_{2}, b_{2}, \eta_{2}$ defined as in DG92:

$$
\begin{aligned}
& u_{2}=\frac{\phi_{22}}{r^{2} \bar{\Omega}^{2}}+\frac{1}{3}\left(1+\eta_{2}\right) \\
& b_{2}=\frac{1}{3} r \frac{\mathrm{d} \eta_{2}}{\mathrm{~d} r} \\
& \eta_{2}=\left(1+\eta_{0}\right)^{2}-1 .
\end{aligned}
$$

\section{B.1. High radial order $p$ modes}

For high radial order $p$ modes, the horizontal component of the fluid displacement is much smaller than the vertical one and $\left|y_{01}\right| \gg\left|z_{0}\right|$. The modes are concentrated toward the surface and $y_{03}, y_{04} \sim 0, U \sim 0, C \sim 1, \lambda \sim V_{g} y_{01}$. Then Eq. (B.8) becomes:

$$
\begin{aligned}
\mathcal{I}_{\mathrm{c}} & \sim \frac{1}{2 I_{0}} \int_{0}^{R} y_{01}^{2}\left\{C \sigma _ { 0 } ^ { 2 } \left[\left(2\left(r \frac{\mathrm{d} u_{2}}{\mathrm{~d} r}+4 u_{2}\right)\right]\right.\right. \\
& \left.-\left(C \sigma_{0}^{2}+3\right) b_{2}-r \frac{\mathrm{d} b_{2}}{\mathrm{~d} r}\right\} \rho_{0} r^{4} \mathrm{~d} r
\end{aligned}
$$

For large $\sigma_{0}$, one further has

$$
\mathcal{I}_{\mathrm{c}} \sim \sigma_{0}^{2} \frac{1}{I_{0}} \int_{0}^{R} y_{01}^{2}\left\{\left(r \frac{\mathrm{d} u_{2}}{\mathrm{~d} r}+4 u_{2}-\frac{b_{2}}{2}\right\} \rho_{0} r^{4} \mathrm{~d} r\right.
$$

As

$I_{0} \sim \int_{0}^{R} y_{01}^{2} \rho_{0} r^{4} \mathrm{~d} r$

then

$\mathcal{I}_{\mathrm{c}} \sim \sigma_{0}^{2}\left\langle\mathcal{S}_{2}\right\rangle$

with

$\left\langle\mathcal{S}_{2}\right\rangle=\left\langle\left(r \frac{\mathrm{d} u_{2}}{\mathrm{~d} r}+4 u_{2}-b_{2}\right)\right\rangle$,

where \langle\rangle is a weighted average defined in Eq. (45). Hence $\mathcal{I}_{\mathrm{c}}$ (thereby $X_{2}, Y_{2}$ ) increases with $\sigma_{0}^{2}$ i.e. with increasing frequency.

\section{B.2. Radial modes}

For radial modes, $\ell=0$, one has $X_{2}=0$ Eq. (9) simplifies as:

$\omega_{2}=\frac{\bar{\Omega}^{2}}{I_{0} \omega_{0}} \int_{0}^{R} y_{01}^{2}\left(b_{2}+\frac{4}{3}\left(1+\eta_{0}\right)\right) \rho_{0} r^{4} \mathrm{~d} r$.

\section{Appendix C: First- and second-order corrections for near-degenerate frequencies}

The different terms which contribute to the first- and secondorder frequency corrections in presence of degeneracy are collected in the $\mu_{j}$ and $\mu_{j k}$ given by Eq. (25). These contributions are:

$$
\begin{aligned}
\omega_{1, a b} & =-\frac{1}{I_{a}}\left\langle\boldsymbol{\xi}_{0, a}|\mathcal{K}| \boldsymbol{\xi}_{0, b}\right\rangle \\
\omega_{2, a b}^{\mathrm{PT}} & =-\frac{1}{I_{a}}\left[\left\langle\boldsymbol{\xi}_{0, a}|\mathcal{K}| \boldsymbol{\xi}_{1, \mathrm{~b}}^{\mathrm{P}}\right\rangle+\left\langle\boldsymbol{\xi}_{0, a}|\mathcal{K}| \boldsymbol{\xi}_{1, \mathrm{~b}}^{\mathrm{T}}\right\rangle\right] \\
& =\omega_{2, a b}^{\mathrm{P}}+\omega_{2, a b}^{\mathrm{T}} \\
\omega_{2, a b}^{\mathrm{D}} & =\frac{1}{2 \bar{\omega}_{0} I_{a}}\left\langle\boldsymbol{\xi}_{0, a}\left|\frac{1}{\rho_{0}}\left(\mathcal{L}_{2}-\rho_{2} \bar{\omega}_{0}^{2}\right)\right| \boldsymbol{\xi}_{0, b}\right\rangle \\
\omega_{2, a b}^{\mathrm{I}} & =\frac{1}{2 \bar{\omega}_{0} I_{a}}\left[\left\langle\boldsymbol{\xi}_{0, a}\left|m^{2} \boldsymbol{\Omega}^{2}-2 m \boldsymbol{\Omega}\right| \boldsymbol{\xi}_{0, b}\right\rangle\right],
\end{aligned}
$$


where $I_{a}$ (Eq. (11)) is the normalization term for the mode $a$,

$$
I_{a}=\left\langle\boldsymbol{\xi}_{0, a} \mid \boldsymbol{\xi}_{0, a}\right\rangle
$$

where the scalar product definition is defined as in Eq. (12) of DG92. $\boldsymbol{\xi}_{1, k}^{P}$ and $\boldsymbol{\xi}_{1, k}^{T}$ represent respectively the poloidal and toroidal components of first-order eigenfunction correction (see Eq. (26) in SGD98 for explicit expressions). The operator $\mathcal{K}$ is defined as:

$\mathcal{K}=m \boldsymbol{\Omega}-\mathrm{i} \boldsymbol{\Omega} \boldsymbol{e}_{z} \times$

Definitions of the operators $\mathcal{L}_{2}, \rho_{2}$ can be found in DG92 and SGD98.

\section{C.1. Detailed expression for $\omega_{1, a b}$}

The use of the definitions of $\mathcal{L}_{2}$ and $\mathcal{K}$ on Eqs. (C.1)-(C.3) yields the following expanded expressions:

$$
\begin{aligned}
\omega_{1, a b}= & \frac{m \bar{\Omega}}{I_{a}} \delta_{\ell_{a} \ell_{b}} \int_{0}^{\mathrm{R}} r^{4} \rho_{0} \mathrm{~d} r\left(1+\eta_{0}\right)\left\{-\left(y_{01, \mathrm{a}} y_{01, \mathrm{~b}}+\Lambda_{b} z_{0, a} z_{0, b}\right)\right. \\
& \left.+\left(y_{01, \mathrm{a}} z_{0, b}+z_{0, a} y_{01, \mathrm{~b}}+z_{0, a} z_{0, b}\right)\right\}
\end{aligned}
$$

where $\delta_{\ell_{a} \ell_{b}}$ is the Kronecker symbol.

\section{C.2. Detailed expression for $\omega_{2, a b}^{P T}$ and $\omega_{2, a b}^{l}$}

In the case of $\omega_{2, a b}^{\mathrm{PT}}$, its components are expressed as:

$$
\begin{aligned}
\omega_{2, a b}^{\mathrm{P}}= & -2 \frac{m^{2} \bar{\Omega}^{2}}{I_{a} \bar{\omega}_{0}} \delta_{\ell_{a} \ell_{b}} \int_{0}^{\mathrm{R}} r^{4} \rho_{0} \mathrm{~d} r\left(1+\eta_{0}\right)^{2}\left\{y_{01, a} y_{1 \mathrm{~b}}\right. \\
& +\Lambda_{a} z_{01, a} z_{1 \mathrm{~b}} \\
& \left.-\left(y_{01, a} z_{1 \mathrm{~b}}+z_{01, a} y_{1 \mathrm{~b}}+z_{01, a} z_{1 \mathrm{~b}}\right)\right\}
\end{aligned}
$$

and

$$
\begin{aligned}
\omega_{2, a b}^{\mathrm{T}}= & \frac{2 \bar{\Omega}^{2}}{I_{a} \bar{\omega}_{0}} \int_{0}^{\mathrm{R}} r^{4} \rho_{0} \mathrm{~d} r\left(1+\eta_{0}\right)^{2}\left\{\delta _ { \ell _ { a } , \ell _ { b } } \left(\tau_{b+1} \tau_{b+1} \Lambda_{\ell_{b}+1}\right.\right. \\
& \left.+\hat{\tau}_{b-1} \hat{\tau}_{b-1} \Lambda_{\ell_{b}-1}\right) \\
& \left.+\delta_{\ell_{a} \ell_{b+2}}\left(\tau_{b+1} \hat{\tau}_{b+1} \Lambda_{b+1}\right)+\delta_{\ell_{a} \ell_{b-2}}\left(\tau_{b-1} \hat{\tau}_{b-1} \Lambda_{b-1}\right)\right\},
\end{aligned}
$$

where $\tau_{b+1}$ and $\hat{\tau}_{b-1}$ are defined as follows:

$$
\begin{aligned}
\tau_{b+1} & =-\frac{\mathrm{i}}{\left(\ell_{b}+1\right)}\left(y_{01, \mathrm{~b}}-\ell_{b} z_{0, b}\right) \gamma_{b+1} \\
\hat{\tau}_{b-1} & =\frac{\mathrm{i}}{\ell_{b}}\left(y_{01, \mathrm{~b}}-\left(\ell_{b}+1\right) z_{0, b}\right) \gamma_{b} .
\end{aligned}
$$

Finally, the inertial term of the correction is given by

$$
\begin{aligned}
\omega_{2, a b}^{\mathrm{I}}= & -m^{2} \frac{\bar{\Omega}^{2}}{2 I_{a} \bar{\omega}_{0}} \delta_{\ell_{a} \ell_{b}} \int_{0}^{\mathrm{R}} r^{4} \rho_{0} \mathrm{~d} r\left(1+\eta_{0}\right)^{2} \\
& \times\left\{\left(y_{01, \mathrm{a}} y_{01, \mathrm{~b}}+\Lambda_{b+1} z_{0, a} z_{0, b}\right)\right. \\
& \left.-2\left(y_{01, \mathrm{a}} z_{0, b}+y_{01, \mathrm{~b}} z_{0, a}+z_{0, a} z_{0, b}\right)\right\} .
\end{aligned}
$$

\section{C.3. Detailed expression for $\omega_{2, a b}^{D}$}

For the correction $\omega_{2, a b}^{\mathrm{D}}$, the angular dependence can be factorized as follows:

$\omega_{2, a b}^{\mathrm{D}}=\frac{\bar{\Omega}^{2}}{\bar{\omega}_{0}} Q_{2 a b} \mathcal{Z}_{a b}$,

where $\boldsymbol{Q}_{2 a b}$ and $\mathcal{Z}_{a b}$ represent the angular and radial components respectively. Selection rules then exist and are imposed by:

$Q_{2 a b}=\int_{\underline{\Omega}} Y_{a}^{*} Y_{b} P_{2}(\cos \theta) \mathrm{d} \underline{\Omega}$,

where $\mathrm{d} \Omega=\sin \theta \mathrm{d} \theta \mathrm{d} \varphi$ is the elementary solid angle and $Y_{b}$ is the spherical harmonics $Y_{\ell_{b}, m_{b}}$. Using the second-order Legendre polynomial:

$P_{2}(\cos \theta)=\frac{3}{2} \cos ^{2} \theta-\frac{1}{2}$,

we obtain

$\begin{aligned} Q_{2 a b}= & \frac{3}{2}\left[\left(\gamma_{a+1}^{2}+\gamma_{a}^{2}-\frac{1}{3}\right) \delta_{a b}\right. \\ & \left.+\gamma_{a} \gamma_{b+1} \delta_{a b+2}+\gamma_{b} \gamma_{a+1} \delta_{a b-2}\right],\end{aligned}$

with

$\gamma_{b}=\sqrt{\mathcal{F}_{b}} \quad$ and $\quad \mathcal{F}_{\ell}=\frac{\ell^{2}-m^{2}}{4 \ell^{2}-1}$.

On the other hand, the radial component $\mathcal{Z}_{a b}$ can be written as SGD98:

$\mathcal{Z}_{a b}=\frac{1}{2 I_{a}} \int_{0}^{\mathrm{R}} r^{2} \rho_{0} \mathrm{~d} r\left\{d_{1} E_{1}+d_{2} E_{2}+r^{2} b_{2} E_{3}+r^{2} b_{3} E_{4}\right\}$,

where

$d_{1}=r^{2} u_{2} \quad d_{2}=r \frac{\mathrm{d} d_{1}}{\mathrm{~d} r} \quad b_{3}=\frac{1}{3} r^{2} \frac{\mathrm{d}^{2} \eta_{2}}{\mathrm{~d} r^{2}}$,

with $b_{2}$ defined in $\mathrm{B} 9$. The non-degenerate expression is a particular case when $a=b$ and it can be found in DG92.

The explicit expressions of $E_{1}, E_{2}, E_{3}$ and $E_{4}$ are given below. For the sake of shortness, the following nomenclature is used in DG92:

$y \equiv y_{01} \quad v \equiv y_{03} \quad W \equiv y_{04}$,

and the following short expressions have been defined:

$q_{j}=y_{j}\left(C \sigma_{0, j}^{2}+4-U\right)-\Lambda_{j} z_{0, j}-W_{j}$

$\lambda_{j}=V_{\mathrm{g}}\left(y_{j}-y_{02, j}+v_{j}\right)$

$s_{j}=\frac{p \Gamma_{1} \lambda_{j}}{r \rho g_{\mathrm{eff}}}$.

for $j=a, b$. We then have:

$E_{k}=E_{k, a b}+E_{k, b a} \quad k=1,4$ 
with

$$
\begin{aligned}
E_{1 a b}= & -\frac{3}{2} q_{a} y_{b}+\frac{1}{2} y_{a} y_{b} \mathcal{F}_{1}-q_{a} \lambda_{b}-\frac{1}{2} y_{b} s_{a} \psi \\
& +\left(C \sigma_{0, a}^{2}+4\right) y_{a} \lambda_{b}-\lambda_{b} W_{a} \\
& +\left(U-\frac{3}{2}\right) W_{a} y_{b}-\Lambda_{a} v_{a} y_{b} \\
& -\Lambda_{a} z_{0, a} W_{b}+3 q_{a} z_{0, b}+\frac{1}{2} z_{0, a} z_{0, b} \mathcal{F}_{3} \\
& -\Lambda_{a} z_{0, a} y_{b}\left(\frac{3}{2}-C \sigma_{0, a}^{2}+U-4\right)-\Lambda_{a} z_{0, a} \lambda_{b} \\
& -z_{0, a} y_{b} \mathcal{F}_{2 b}+z_{0, a} y_{02, b}\left(\Lambda_{a b}^{+}-3\right)(U-4) \\
& +\frac{1}{2}(4-U)\left(y_{a} q_{b}-s_{a} \Lambda_{b} z_{0 b}\right),
\end{aligned}
$$$$
E_{2 a b}=\frac{1}{2}\left(C \sigma_{0, a}^{2}-U\right) y_{a} y_{b}+\frac{1}{2} y_{a} q_{b}+(U-2) y_{a} s_{b}
$$$$
-y_{b} W_{a}+\frac{1}{2}\left(\Lambda_{a b}^{+}-3\right) C \sigma_{0, a}^{2} z_{0, a} z_{0, b}+\Lambda_{b} z_{0 b} y_{a}
$$$$
-\left(\Lambda_{a b}^{+}-3\right)\left(z_{0, b} y_{02, a}\right)-\frac{1}{2} s_{a} \Lambda_{b} z_{0 b}
$$$$
E_{3 a b}=-\frac{1}{2} C \sigma_{0, a}^{2}\left(y_{a} y_{b}+\left(\Lambda_{a b}^{+}-3\right) z_{0, a} z_{0, b}\right)
$$$$
-\frac{1}{2} y_{a} s_{b}(U+6)-3 y_{b} z_{0, a}-\frac{1}{2}\left(\Lambda_{a}-\Lambda_{b}\right) y_{b} z_{0, a}
$$$$
-\left(3-\Lambda_{a b}^{+}\right) z_{0, a} v_{b}+y_{a} W_{b}
$$$$
+\frac{1}{2} s_{a} \lambda_{b}\left(\frac{\partial \ln \Gamma_{1}}{\partial \ln r}\right)_{p_{0}},
$$

$E_{4}=-\frac{1}{2}\left(y_{a} s_{b}+y_{b} s_{a}\right)$,

with

$$
\begin{aligned}
\mathcal{F}_{1} & =6+U\left(U-2\left(A^{*}+V_{\mathrm{g}}\right)-3\right)+(3-U) C \sigma_{0, a}^{2} \\
\mathcal{F}_{2 a} & =\left(\Lambda_{a b}^{+}-3\right)(U-4)+\Lambda_{a b}^{+}\left(C \sigma_{0, a}^{2}+4-U\right) \\
\mathcal{F}_{3} & =\left(\Lambda_{a b}^{+}-3\right) C \sigma_{0, a}^{2}(6-U)+6 \Lambda_{a b}^{+} \\
\psi & =(1-U)(4-U)+6+U(U-3) \\
\Lambda_{a b}^{+} & =\frac{1}{2}\left(\Lambda_{a}+\Lambda_{b}\right) \\
\Lambda_{b a}^{-} & =\frac{1}{2}\left(\Lambda_{b}-\Lambda_{a}\right)=-\Lambda_{a b}^{-},
\end{aligned}
$$

where

$\Lambda_{j}=\ell_{j}\left(\ell_{j}+1\right)$,

with $j$ representing the modes $a$ and $b$.

\section{C.3.1. High radial order $p$ modes}

Following the same approximations for modes concentrated towards the surface as in Sect. B.1, and using the following approximations (from the definitions Eqs. (C.22)-(C.24))

$q_{j} \sim y_{j}\left(C \sigma_{0, j}^{2}+4\right), \lambda_{j} \sim V_{\mathrm{g}} y_{j}, s_{j} \sim y_{j} \quad j=a, b$.

Equation (C.19) becomes for large $\sigma_{0}$ :

$\mathcal{Z}_{a b} \sim \frac{1}{I_{a}} \int_{0}^{\mathrm{R}} C \sigma_{0}^{2}\left[4 u_{2}+r \frac{d u_{2}}{d r}-\frac{b_{2}}{2}\right] y_{a} y_{b} \rho_{0} r^{4} \mathrm{~d} r$,

where we have used definitions given in Eqs. (B.9) and (C.20). This expression is the same as the one in $\mathrm{B} 11$ when $a=b$.

Using the definition of weighted average given in Eq. (45) with $y_{a} y_{b}$ instead of $y_{01}^{2}$, Eq. (C.34) can be rewritten as :

$\mathcal{Z}_{a b} \sim \sigma_{0}^{2}\left\langle\mathcal{S}_{2}\right\rangle_{a b}$

Finally, $\omega_{2, a b}^{\mathrm{D}}($ Eq. $(\mathrm{C} 14))$ for high radial order $p$ modes can be written as

$\omega_{2, a b}^{\mathrm{D}} \sim \frac{\bar{\Omega}^{2}}{G M / R^{3}} Q_{2 a b} \bar{\omega}_{0}\left\langle\mathcal{S}_{2}\right\rangle_{a b}$. 